\title{
Computational aspects of morphological instabilities using isogeometric analysis
}

\author{
Berkin Dortdivanlioglu, Ali Javili, Christian Linder* \\ Department of Civil and Environmental Engineering, Stanford University, Stanford, CA 94305, USA
}

\begin{abstract}
Morphological instabilities play a crucial role in the behavior of living systems as well as advanced engineering applications. Such instabilities initiate when a thin stiff film on a compliant substrate is subject to compressive stresses. For bilayer systems, the first mode of instability is sinusoidal wrinkling. While the critical conditions to induce wrinkling are extensively studied, the more complex patterns formed beyond wrinkling remain elusive and poorly understood. The objective of this contribution is to establish a generic computational framework capable of capturing various instabilities, using isogeometric analysis (IGA) enhanced with a concurrent eigenvalue analysis. It is shown that the eigenvalue analysis provides quantitatively accurate predictions for the onset of instabilities. In addition, the results are compared with the standard finite element analysis (FEA) and it is clearly observed that IGA furnishes significantly more accurate results compared to FEA, for the same number of degrees of freedom. We believe that this generic framework is widely applicable to advance our understanding of emergence and evolution of morphological instabilities for a rich variety of applications in soft materials and living systems.
\end{abstract}

Keywords: Morphological instabilities, Isogeometric analysis, Wrinkling, Period-doubling, Eigenvalue analysis

\section{Introduction}

Morphological instabilities due to compressive stresses in materials lead to various folding patterns with distinctive characteristics and have numerous recent applications, see e.g. [1-4] and references therein. Understanding morphological instabilities has attracted increasing attention over the past years, since not only does it help to explain the morphogenesis in living systems, but it also serves as a basis for design of advanced engineering systems [5-9]. Common examples of instabilities during morphogenesis of living systems include the cortical folding in the brain [10-13], wrinkling of skin [14, 15], folding of asthmatic airways [16, 17], and channel formation in biofilms [18, 19]. Malformation of such instabilities can signal abnormal evolution of the tissue, often related to fatal diseases. Furthermore, harnessing instabilities in advanced engineering systems paves the way towards stretchable electronics, tunable adhesive property, material parameter characterization, self-organized surface patterns, smart morphable surfaces in aerodynamics, drug delivery, and understanding geological folded structures [20-23], to name few.

The objective of this manuscript is to develop an accurate and reliable computational framework suitable to study morphological instabilities using the isogeometric analysis (IGA) pioneered by Hughes et al. [24]. Available numerical studies on this topic to date are limited to the classical finite element analysis (FEA) and suffer from the intrinsic limitations of FEA as compared to IGA. Therefore, the two main ingredients of the work presented here are $(i)$ growthinduced instabilities and (ii) isogeometric analysis. A brief review of these topics is next given in Sec. 1.1 and Sec. 1.2 , respectively.

\subsection{State of the art review of growth-induced instabilities}

When a thin film adhered to a compliant substrate is subject to compression beyond a critical strain, wrinkles form to release the energy. The critical conditions for the onset of wrinkling and the resulting morphological patterns

${ }^{*}$ Corresponding author. Email address: linder@stanford.edu 
have extensively been exploited via theoretical, experimental and numerical contributions, see for instance $[1,25,26]$ among others. In particular, pioneering works of Biot [27] and Allen [28] propose analytical solutions to the linear instability problem of a thin film adhered to a compliant infinite half-space. These solutions prove to be useful in aforementioned engineering applications as long as the strains in the substrate remain small, which is the case until the onset of wrinkling. Recent works [e.g. 29-32] also consider highly nonlinear behavior of the film and substrate and conduct extensive numerical analyses for investigating the critical conditions and the influence of the substrate prestretch on the onset of wrinkling for jointly compressed film-substrate systems. Further increase in compression beyond the onset of wrinkling, evolves the wrinkles into higher instability modes such as crease, perioddoubling, localized folds, and ridges [3, 33-37]. Typically, the transition from wrinkling to period-doubling occurs for systems with a stiff thin film adhered to a compliant substrate, and is followed by a period-quadrupling and later on, localized folding $[38,39]$. This transition has recently been observed in experiments $[38,40]$ and described by approximate theoretical work of Brau et al. [38]. Furthermore, numerical models [31, 40-42] provide a deeper insight into the mechanics of period-doubling. The critical conditions for the onset and evolution of the perioddoubling instability largely depend on the nonlinear behavior of substrate [38]. Besides certain model non-linearities, Poisson's ratio and substrate prestretch play an important role in triggering period-doubling since they provoke the material nonlinearities [31, 34, 40, 42, 43]. The higher instability modes often emerge at large strain levels where both material and geometrical non-linearities in the substrate are prevailing. Therefore, the analytical approaches to study the critical conditions for the transition from wrinkling are limited to substantially simplified cases with a limited range of material parameters [37, 44]. Hence, it is crucial to establish an accurate and reliable numerical framework capable of capturing higher order instability modes such as period-doubling. This task shapes the foundations of this manuscript and we develop a computational framework to study morphological instabilities using IGA.

\subsection{State of the art review of isogeometric analysis}

Isogeometric analysis has recently been proposed as a numerical method to bridge the gap between computer aided design (CAD) and analysis [24] and has been further extended by Cottrell et al. [45] and Bazilevs et al. [46] among others. The main idea of IGA is to employ the same basis functions of the geometric parametrization of CAD technology such as B-splines and Non-uniform Rational Bezier Spline (NURBS) to solve partial differential equations. The ability to construct the exact computational geometry is due to the high continuity of NURBS elements. This outstanding feature also eases the use of higher order basis functions in the analysis process [47]. The exact representation of the computational domain is valid throughout the refinement process from the coarsest to the finest discretization with the converged solution [48]. However, the tensor product generation of the parametric space hinders the refinement flexibility over the computational domain $[46,49]$. As a remedy to this geometric limitation, new methods permitting the local refinement have been proposed such as suitable T-splines [50-54] or hierarchical and local refinement of splines [49,55-57]. Despite all the recent advances, this issue has remained as an active research area [58, 59]. Furthermore, IGA has attracted an increasing attention, not only due to its capability to accurately represent the computational domain, but it also exhibits superior performance in approximating the solution as compared to the standard FEA [60-63]. These unique features of IGA prove to be highly beneficial for free-form simulation of living systems and flexible design of novel engineering systems $[60,61,64,65]$. Isogeometric analysis is a mature field and has been explored for various applications [64, 66-78].

The main purpose of this contribution is to formulate a computational framework using IGA to study the morphological instabilities in basic bilayer systems with a stiff thin film adhered to a compliant substrate. The eigenvalue analysis approach of Javili et al. [79] is adopted to compute the onset of instabilities. This methodology is relatively straightforward and very efficient in the current context since the eigenvalue approach [79] is accurate and inherently compatible with IGA. Equipped with an accurate framework capable of predicting critical conditions, we then compare the performance of IGA against FEA. Our numerical analysis clearly shows that IGA furnishes more accurate results for the same number of degrees of freedom compared to FEA.

This manuscript is organized as follows. Section 2 deals with the formulation of the problem and our methodology. The kinematics of the finite growth is concisely introduced in Sec. 2.1 followed by governing equations for growing continua within the finite deformation setting in Sec. 2.2. Isogeometric analysis is briefly reviewed in Sec. 2.3. Using IGA, we perform an exhaustive numerical investigation to fully understand the nature of the critical conditions for primary and secondary instabilities in Sec. 3. First, we devise a systematic study and clearly highlight the superior performance of IGA against FEM for growth-induced instability problems. Furthermore, our analysis reveals a 
quantitative prediction of the critical conditions for period-doubling via eigenvalue analysis. We explore the critical conditions for instabilities for a broad range of stiffness ratios and gather all the results in a phase diagram. This provides a significant insight into the design of bilayer systems in light of their instability modes such as wrinkling or period-doubling. Section 4 concludes this work and provides further outlook and possible extensions.

\section{Problem formulation}

The objective of this manuscript is to carry out a comprehensive computational study on morphological instabilities due to compressive stresses in the material. The potential sources of compressive stresses that can results in morphological instabilities are mechanical loading, confined thermal loading and differential material growth (atrophy) or swelling (shrinkage). Henceforth, we adopt the concept of finite growth within a confined domain [80] as the driving mechanism to induce the residual stresses in the body and eventually resulting in instabilities.

\subsection{Kinematics of finite growth}

In order to formulate the kinematics of finite growth, we start from the continuum mechanics at finite deformation. Consider a continuum body that occupies the reference configuration $\mathcal{B}_{0}$ at time $t=0$. The non-linear orientationpreserving deformation map $\varphi$ relates the reference placement $X$ to the current placement $x=\varphi(X, t)$ at any time $t>0$. The corresponding linear map from the reference configuration $\mathcal{B}_{0}$ to the current configuration $\mathcal{B}_{t}$ is the deformation gradient defined as $\boldsymbol{F}:=\operatorname{Grad} \varphi$. The deformation gradient $\boldsymbol{F}$ maps the infinitesimal line element $\mathrm{d} \boldsymbol{X}$ in the reference configuration to its counterpart $\mathrm{d} \boldsymbol{x}$ in the current configuration according to $\mathrm{d} \boldsymbol{x}=\boldsymbol{F} \mathrm{d} \boldsymbol{X}$. The Jacobian of the deformation gradient, $J:=\operatorname{Det} \boldsymbol{F}=\mathrm{d} v / \mathrm{d} V$, yields the ratio of the volume element $\mathrm{d} v$ due to the deformation to its reference counterpart $\mathrm{d} V$. The common approach to model volumetric growth is the multiplicative decomposition of the deformation gradient $[80-86]$ as

$$
\boldsymbol{F}=\boldsymbol{F}_{e} \boldsymbol{F}_{g} \quad \Rightarrow \quad \boldsymbol{F}_{e}=\boldsymbol{F}_{g}{ }^{-1} \quad \text { and } \quad J_{e}=\operatorname{det} \boldsymbol{F}_{e}, \quad J_{g}=\operatorname{det} \boldsymbol{F}_{g}
$$

into its elastic and growth parts $\boldsymbol{F}_{e}$ and $\boldsymbol{F}_{g}$, respectively, as illustrated in Fig. 1. The growth part $\boldsymbol{F}_{g}$ linearly maps the stress-free reference configuration $\mathcal{B}_{0}$ to the stress-free grown configuration $\mathcal{B}_{g}$. In general, this grown configuration $\mathcal{B}_{g}$ and the map $\boldsymbol{F}_{g}$ are incompatible. The elastic part $\boldsymbol{F}_{e}$ maps this grown configuration back to a compatible and stressed configuration $\mathcal{B}_{t}$.

We consider hereinafter only the volumetric and homogeneous growth that is independent of the deformation. To model a volumetric growth in a preferred direction $\boldsymbol{N}_{g}$, for example an anisotropic growth, the growth tensor $\boldsymbol{F}_{g}$ is

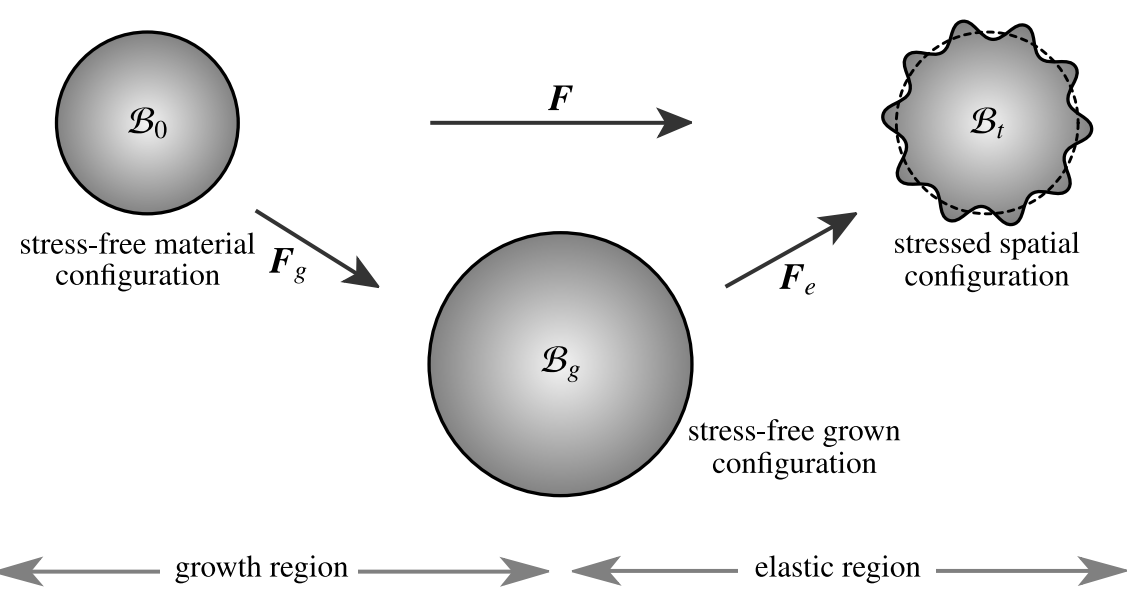

Figure 1: Illustration of kinematics of finite growth based on the multiplicative decomposition of the deformation gradient $\boldsymbol{F}$ into an elastic part $\boldsymbol{F}_{e}$ and a growth part $\boldsymbol{F}_{g}$. The intermediate configuration $\mathcal{B}_{g}$ is referred to as incompatible stress-free grown configuration. 
defined as

$$
\boldsymbol{F}_{g}=\boldsymbol{I}+g\left(\boldsymbol{I}-\boldsymbol{N}_{g} \otimes \boldsymbol{N}_{g}\right)=(1+g) \boldsymbol{I}-g \boldsymbol{N}_{\boldsymbol{g}} \otimes \boldsymbol{N}_{g}
$$

in which $\boldsymbol{I}$ denotes the invariant identity tensor and $g$ is the scalar-valued growth multiplier. Using (2), we define a transversely isotropic growth such that the material grows in the plane with the unit normal $N_{g}$ but it does not grow along the direction $N_{g}$. In the absence of a preferred direction, we recover the isotropic growth tensor, where the material grows freely in all directions, as

$$
\boldsymbol{F}_{g}=(1+g) \boldsymbol{I}
$$

For further details on growth and remodeling, see for instance [84, 87] and references therein.

\subsection{Governing equations for growing continua}

This section briefly lists the governing equations for a continuum body that undergoes growth and finite deformation. The governing equations of the problem of interest here are the balances of linear and angular momentum as well as the thermodynamically consistent constitutive laws to prescribe the material behavior.

For the sake of simplicity, we formulate the governing equations in a Lagrangian setting and with respect to the quantities associated with the reference configuration. In a quasi-static process, the balance of linear momentum in the domain $\mathcal{B}_{0}$ reads

$$
\operatorname{Div} \boldsymbol{P}+\boldsymbol{b}_{0}=\mathbf{0} \quad \text { in } \mathcal{B}_{0} \quad \text { subject to } \quad \boldsymbol{P} \boldsymbol{N}=\boldsymbol{t}_{0} \quad \text { on } \partial \mathcal{B}_{0}
$$

in which the divergence operator $\operatorname{Div}\{\bullet\}=\operatorname{Grad}\{\bullet\}: \boldsymbol{I}$ is the trace of the gradient defined as $\operatorname{Grad}\{\bullet\}=\partial\{\bullet\} / \partial \boldsymbol{X}$. Clearly, both the divergence and the gradient operators here are defined with respect to the reference configuration. The Piola stress is denoted $\boldsymbol{P}$ and $\boldsymbol{b}_{0}$ is the body force density in $\mathcal{B}_{0}$. On the boundary of the domain $\partial \mathcal{B}_{0}$, the externally prescribed traction is denoted $\boldsymbol{t}_{0}$. The balance of the angular momentum in the reference configuration reads

$$
\boldsymbol{P} \boldsymbol{F}^{\mathrm{T}}=\boldsymbol{F} \boldsymbol{P}^{\mathrm{T}}
$$

which equivalently corresponds to the symmetry of Cauchy stresses. Without going into too much details, it should be mentioned that we a priori satisfy the balance of angular momentum through material frame indifference of the free energy. For the constitutive equation we employ an isotropic elastic response for the material and introduce the Helmholtz free energy as

$$
\psi=\psi\left(\boldsymbol{F}, \boldsymbol{F}_{g}\right)=\psi_{e}\left(\boldsymbol{F}_{e}\right)
$$

Making use of the second law of thermodynamics and the Coleman-Noll procedure, we can relate the Piola stress to the free energy density in the reference configuration by

$$
\boldsymbol{P}:=\frac{\partial \psi}{\partial \boldsymbol{F}}=\frac{\partial \psi_{e}}{\partial \boldsymbol{F}_{e}}: \frac{\partial \boldsymbol{F}_{e}}{\partial \boldsymbol{F}}=\frac{\partial \psi_{e}}{\partial \boldsymbol{F}_{e}}:\left(\boldsymbol{I} \bar{\otimes} \boldsymbol{F}_{g}^{-\mathrm{T}}\right)=\boldsymbol{P}_{e} \boldsymbol{F}_{g}^{-\mathrm{T}} \quad \text { with } \quad \boldsymbol{P}_{e}:=\frac{\partial \psi_{e}}{\partial \boldsymbol{F}_{e}} .
$$

Note that the non-standard tensor product $\bar{\otimes}$ of two arbitrary second order tensors $\boldsymbol{A}$ and $\boldsymbol{B}$ in index notation refers to $[\boldsymbol{A} \bar{\otimes} \boldsymbol{B}]_{i j k l}=[\boldsymbol{A}]_{i k}[\boldsymbol{B}]_{j l}$. The tangent moduli $\mathrm{A}$ can be derived by taking the second derivative of the free energy with respect to the deformation gradient, resulting in a fourth order tensor, as

$$
\mathbb{A}:=\frac{\mathrm{d}}{\mathrm{d} \boldsymbol{F}}\left(\frac{\partial \psi}{\partial \boldsymbol{F}}\right)=\frac{\mathrm{d} \boldsymbol{P}}{\mathrm{d} \boldsymbol{F}}=\frac{\mathrm{d}\left(\boldsymbol{P}_{e} \boldsymbol{F}_{g}{ }^{-\mathrm{T}}\right)}{\mathrm{d} \boldsymbol{F}_{e}}: \frac{\mathrm{d} \boldsymbol{F}_{e}}{\mathrm{~d} \boldsymbol{F}}=\left(\boldsymbol{I} \bar{\otimes} \boldsymbol{F}_{g}{ }^{-1}\right): \mathbb{A}_{e}:\left(\boldsymbol{I} \otimes \boldsymbol{F}_{g}{ }^{-\mathrm{T}}\right) \quad \text { with } \quad \mathbb{A}_{e}:=\frac{\partial \boldsymbol{P}_{e}}{\partial \boldsymbol{F}_{e}} .
$$

We employ the isotropic neo-Hookean elastic material model and specify the elastic free energy as

$$
\psi_{e}=\frac{1}{2} \mu\left(\boldsymbol{F}_{e}: \boldsymbol{F}_{e}-3-2 \ln J_{e}\right)+\frac{1}{2} \lambda\left[\frac{1}{2}\left(J_{e}^{2}-1\right)-\ln J_{e}\right] \quad \text { with } J_{e}=\operatorname{det} \boldsymbol{F}_{e}
$$


in which $\mu$ and $\lambda$ are the Lamé material parameters, instead of adopting alternative micromechanically motivated material models [88-90]. Then, the corresponding elastic Piola stress $\boldsymbol{P}_{e}$ and the tangent $\boldsymbol{A}_{e}$ are given as

$$
\begin{aligned}
& \boldsymbol{P}_{e}=\frac{\partial \psi_{e}}{\partial \boldsymbol{F}_{e}}=\mu\left(\boldsymbol{F}_{e}-\boldsymbol{F}_{e}^{-\mathrm{T}}\right)+\frac{1}{2} \lambda\left(J_{e}^{2}-1\right) \boldsymbol{F}_{e}^{-\mathrm{T}} \\
& \mathbb{A}_{e}=\frac{\partial \boldsymbol{P}_{e}}{\partial \boldsymbol{F}_{e}}=\mu\left(\mathbb{I}-\mathbb{D}_{e}\right)+\frac{1}{2} \lambda\left(J_{e}^{2}-1\right) \mathbb{D}_{e}+\lambda J_{e}^{2} \boldsymbol{F}_{e}^{-\mathrm{T}} \bar{\otimes} \boldsymbol{F}_{e}^{-\mathrm{T}} \text { with } \mathbb{I}:=\boldsymbol{I} \bar{\otimes} \boldsymbol{I} \text { and } \mathbb{D}_{e}:=-\boldsymbol{F}_{e}^{-\mathrm{T}} \underline{\otimes} \boldsymbol{F}_{e}{ }^{-1} .
\end{aligned}
$$

Here, $\mathbb{I}$ denotes the fourth-order identity tensor and $\mathbb{D}_{e}:=\partial \boldsymbol{F}_{e}^{-\mathrm{T}} / \partial \boldsymbol{F}_{e}=-\boldsymbol{F}_{e}^{-\mathrm{T}} \otimes \boldsymbol{F}_{e}^{-1}$ with $\left[\mathbb{D}_{e}\right]_{i j k l}=-\left[\boldsymbol{F}_{e} e^{-\mathrm{T}}\right]_{i l}\left[\boldsymbol{F}_{e}^{-1}\right]_{j k}$.

In order to carry out the computational implementation within a finite element framework, we require the weak form of the balance equations. The weak form is obtained through a standard procedure of $(i)$ multiplying the strong form of the balance (4) with a test function $\delta \varphi$, (ii) integration over the domain $\mathcal{B}_{0}$ and (iii) applying the divergence theorem. The weak form of the balance of linear momentum reads

$$
\int_{\mathcal{B}_{0}} \boldsymbol{P}: \operatorname{Grad} \delta \boldsymbol{\varphi} \mathrm{d} V-\int_{\mathcal{B}_{0}} \delta \boldsymbol{\varphi} \cdot \boldsymbol{b}_{0} \mathrm{~d} V-\int_{\partial \mathcal{B}_{0}} \delta \boldsymbol{\varphi} \cdot \boldsymbol{t}_{0} \mathrm{~d} A=0 .
$$

Note, the weak form (12) is identical for both FEA and IGA. Only after employing the Galerkin isoparametric concept, FEA and IGA are distinguishable since they accommodate different basis functions. More precisely, IGA uses NURBS and the standard FEA uses Lagrange polynomial basis functions.

\subsection{Isogeometric analysis}

The standard finite element analysis is well-established today and adopts Lagrange polynomials as the basis functions for discretization. The isogeometric analysis, on the other hand, uses NURBS to discretize the space. In this section, we briefly introduce the B-spline and NURBS functions together with their usage in IGA.

Consider a sequence of $n+p+1$ nondecreasing numbers $\Xi=\left\{\xi_{1}, \xi_{2}, \ldots, \xi_{n+p+1}\right\}$ where $p$ is the polynomial order and $n$ is the number of basis functions (and control points). Each $\xi_{i}$ corresponds to a coordinate in the parametric space denoted as a knot. Hence, the knot vector $\Xi$ splits the parametric space into pieces, often referred to as knot spans, that can be viewed as the elements in the standard finite element context. A uniform knot vector can be constructed with equally spaced knots. For a given polynomial order $p$ and knot vector $\Xi$, the B-spline basis functions are constructed recursively starting with the zeroth order basis function. The zeroth order basis function $(p=0)$ is defined with piece-wise constants for each knot span as

$$
N_{i, 0}(\xi)= \begin{cases}1 & \text { if } \quad \xi_{i} \leq \xi<\xi_{i+1} \\ 0 & \text { otherwise }\end{cases}
$$

For $p>0$, the B-spline basis functions are defined by

$$
N_{i, p}(\xi)=\frac{\xi-\xi_{i}}{\xi_{i+p}-\xi_{i}} N_{i, p-1}(\xi)+\frac{\xi_{i+p+1}-\xi}{\xi_{i+p+1}-\xi_{i+1}} N_{i+1, p-1}(\xi) .
$$

This recursive process is referred to as Cox-de Boor recursion formula and various efficient algorithms for their computations exist [91]. Note that the basis functions with $p=1$ are the same as linear finite element functions. The $\mathrm{B}$-spline basis functions with the order of $p$ are $C^{p-1}$ continuous over the knot spans. For a knot repeating itself $k$ times in the knot vector, the basis function at the knot reduces its continuity to $C^{p-k}$. Figure 2 illustrates an example of quadratic B-spline with $\mathrm{p}=2$ basis functions with the knot vector $\Xi=\{0,0,0,1 / 4,1 / 2,3 / 4,1,1,1\}$.

A B-spline curve $\boldsymbol{C}(\xi)$ in $\mathbb{R}^{d}$ is constructed via linear combinations of basis functions $N_{i, p}(\xi)$ with coefficients $\boldsymbol{B}_{i}$ and the polynomial order $p$ as

$$
\boldsymbol{C}(\xi)=\sum_{i=1}^{n} N_{i, p}(\xi) \boldsymbol{B}_{i}
$$



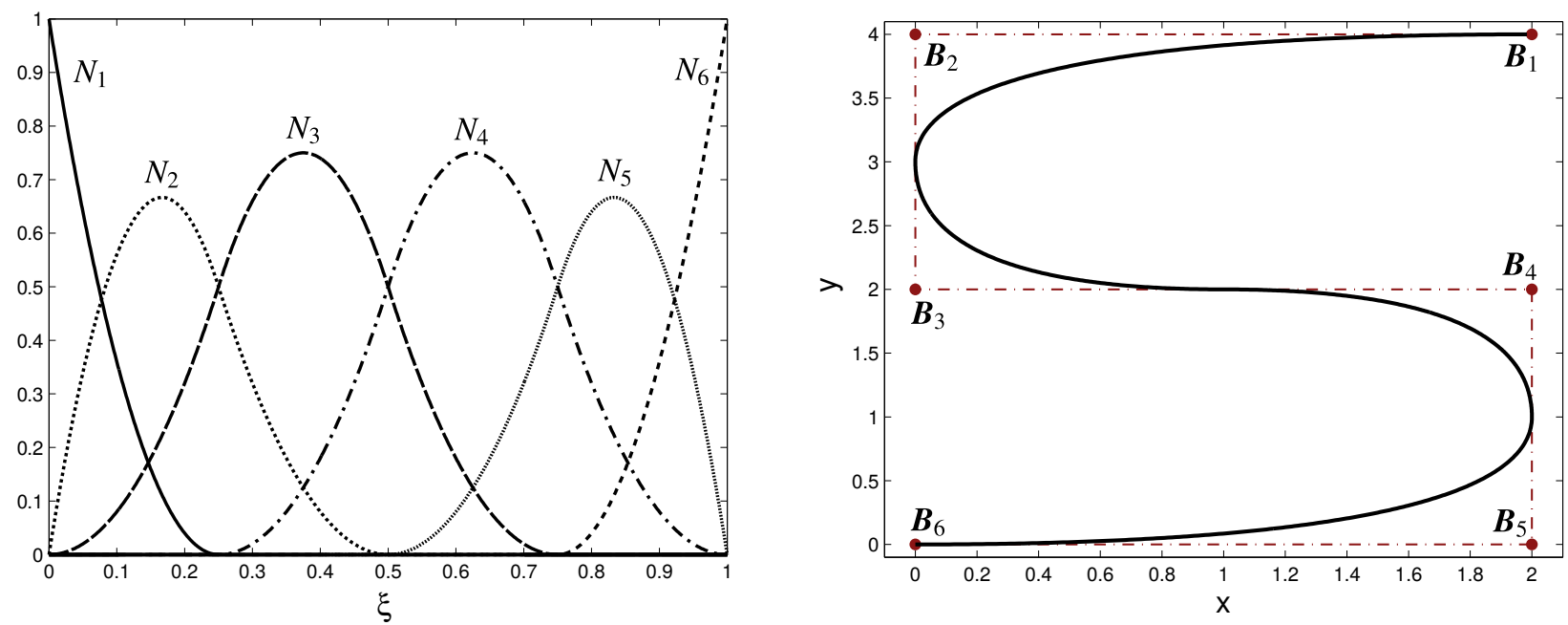

Figure 2: An example of quadratic B-spline with $\mathrm{p}=2$ basis functions with the knot vector $\Xi=\{0,0,0,1 / 4,1 / 2,3 / 4,1,1,1\}$ with interpolatory end and the curve generated from the quadratic B-spline functions and the control points.

where $\boldsymbol{B}$ is the set of control points in $\mathbb{R}^{d}$, referred to as the nodal coordinates in the standard finite element context. However, control points are not interpolated by the B-spline functions, see Fig. 2, and this behavior is one of the major differences with the standard finite element analysis. One dimensional NURBS functions $R_{i, p}$ can be constructed through the B-spline functions (14) using

$$
R_{i}^{p}(\xi)=\frac{N_{i, p}(\xi) w_{i}}{\sum_{i=1}^{n} N_{i, p}(\xi) w_{i}}
$$

where $w_{i}$ is the weight of the corresponding control point $\boldsymbol{B}_{i}$. Two-dimensional surface NURBS functions and threedimensional volume NURBS functions can be derived via the tensor products

$$
R_{i, j}^{p, q}(\xi, \eta)=\frac{N_{i, p}(\xi) N_{j, q}(\eta) w_{i j}}{\sum_{i=1}^{n} \sum_{j=1}^{m} N_{i, p}(\xi) N_{j, q}(\eta) w_{i j}} \quad \text { and } \quad R_{i, j, k}^{p, q, r}(\xi, \eta, \zeta)=\frac{N_{i, p}(\xi) N_{j, q}(\eta) N_{k, r}(\zeta) w_{i j k}}{\sum_{i=1}^{n} \sum_{j=1}^{m} \sum_{k=1}^{l} N_{i, p}(\xi) N_{j, q}(\eta) N_{k, r}(\zeta) w_{i j k}}
$$

respectively, using the knot vectors $\Xi=\left\{\xi_{1}, \xi_{2}, \ldots, \xi_{n+p+1}\right\}, \mathcal{H}=\left\{\eta_{1}, \eta_{2}, \ldots, \eta_{m+q+1}\right\}$ and $\mathcal{Z}=\left\{\zeta_{1}, \zeta_{2}, \ldots, \zeta_{l+r+1}\right\}$.

\section{Numerical examples}

The objective of this section is to understand the underlying mechanism that induces morphological instabilities and to illustrate the capability of IGA to capture both the onset of instabilities as well as the post-buckling behavior via a series of numerical examples. First, we carry out an in-depth buckling analysis on a growing slender beam in Sec. 3.1. This example is particularly chosen due to its simplicity and that it possesses a closed-form analytical solution, presented in Appendix A.1, for further comparisons. We have verified our numerical results against the analytical solution but, we have omitted the details in this presentation so as to remain focused on the main objectives of the manuscript. Next, in Sec. 3.2 we investigate the nature of wrinkling and period-doubling instabilities in bilayer systems. For the wrinkling of bilayer systems, some analytical solutions can be found in the literature [28]. Nevertheless, such analytical solutions are based on various simplifying assumptions such as $(i)$ the substrate being an infinite half-space and (ii) large stiffness ratios between the film and the substrate. The limited applicability of the anaytical solution for wrinkling, justifies the need for a reliable computational framework. More importantly, these analytical solutions are only relevant for the wrinkling but the more interesting folding patterns such as period-doubling, to date, do not possess any analytical solution, in general [38]. 


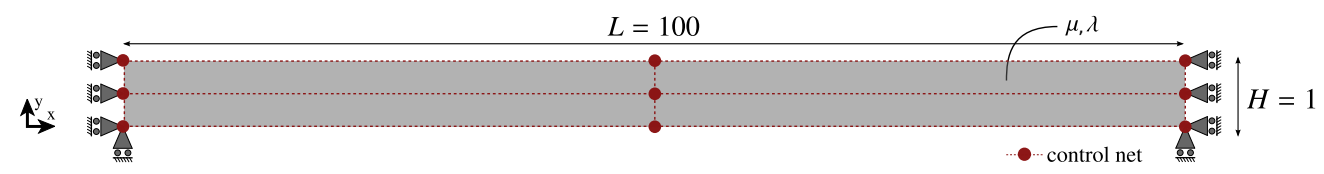

Figure 3: Geometry and boundary conditions of a growing beam.

\subsection{Buckling of a growing slender beam and associated bifurcation tree}

In the first part, we study the buckling analysis of a growing slender beam with the length of $L=100$ and unit cross section under the plane-strain conditions as shown in Fig. 3. The material parameters are the Poisson's ratio $v=0.49$ and the modulus of elasticity $E=10^{5}$. The geometry is constructed using a single patch with knot vectors $\Xi=\{0,0,0,1,1,1\}$ and $\mathcal{H}=\{0,0,0,1,1,1\}$, and the polynomial order of $p=q=2$. The mesh refinement has been performed through knot insertion at desired directions. The beam only grows in the direction of length with the anisotropic growth tensor $\boldsymbol{F}_{g}=\boldsymbol{I}+g \boldsymbol{e}_{x} \otimes \boldsymbol{e}_{x}$. The accuracy of IGA is compared with FEA per degrees of freedom and the results clearly reveal a superior performance of IGA over FEA.

The slender beam in Fig. 3 grows along its length and due to the constraints at its both ends, the growth induces residual stresses in the beam resulting in the instabilities. Adopting the eigenvalue analysis detailed in [79], we solve the problem using IGA and compute the first 8 eigenmodes of the beam together with their corresponding critical growth values. Furthermore, we perform an $h$-adaptive convergence study for all modes. Figure 4 shows both the critical growth and the percent error with respect to the finest solution. Several conclusions can be drawn from the results in Fig. 4. First, increasing the number of elements along the beam improves the accuracy in critical growth and the post buckling shape. Nevertheless, increasing the number of elements across the thickness of the beam does not influence the convergence behavior which would not be the case for linear discretization. Clearly, a single element in the thickness direction is enough for IGA to recover the solutions for all eigenmodes although the bending deformations are significant. As a result of this study, we observed that 12 is the minimum number of quadratic elements to properly capture the $8^{\text {th }}$ eigenmode. We compared both FEA and IGA by computing the buckling modes (eigenvectors) for each mode using only 12 elements along the length in Fig. 5. Due to the inherent characteristic of FEA to approximate the computational geometry through piece-wise polynomials, we observe a more pronounced difference from IGA at higher modes. Therefore, IGA not only preserves the smoothness for all modes due to the high continuity of NURBS elements, but it also obtains the results using less degrees of freedoms compared to the FEA.

We extend our study to capture post-buckling behavior for higher modes and generate the so-called bifurcation trees showing the deformation of the beam for each individual mode. To capture the increase of the amplitude of the buckled beam, we adopt the perturbation technique commonly used for post buckling studies. Clearly, the eigenvalue analysis is not a suitable method to follow the evolution of the growth-induced instabilities while it is extremely useful to predict the onset of the instabilities. Employing the results obtained previously via the eigenvalue analysis, we trigger the desired instability modes via the perturbation technique. The beam is perturbed with a small point load of the magnitude $10^{-4}$ at the middle. Figure 6 illustrates the amplitude of each instability mode up to $8^{\text {th }}$ mode for a growing beam discretized using $100 \times 1$ elements. The amplitude shown in Fig. 6 is the maximum peak amplitude of the waves. We present the even and the odd modes in different graphs since the amplitude refers to different measures in symmetrical and anti-symmetrical modes. The perturbation applied on the structure causes the onset of the instabilities to be slightly earlier compared to eigenvalue analysis (not visible on the graphs). This negligible difference confirms that the applied perturbation has been appropriate for the specific problem at hand. The framework using IGA captures the higher modes accurately and successfully generates the whole bifurcation tree via the perturbation technique supplemented by eigenvalue analysis.

\subsection{Wrinkling instability}

In this section, we study the wrinkling instabilities of the bilayer system depicted in Fig. 7. The bilayer system of interest is a rectangular domain under the plane-strain conditions, constrained on the sides only in the x-direction and at the bottom only in the y-direction. The domain has the length $L$ and is composed of a substrate with the height $H_{s}$ 
1 element across the thickness
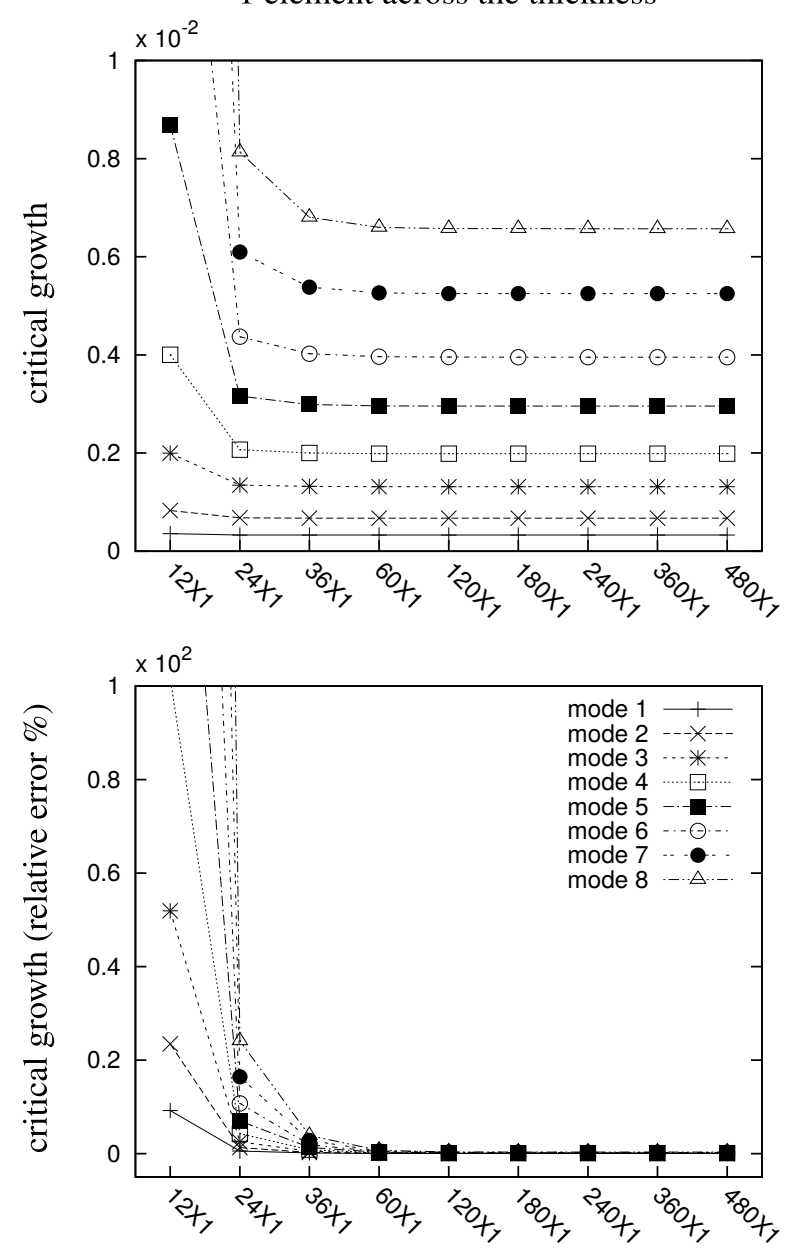

number of elements
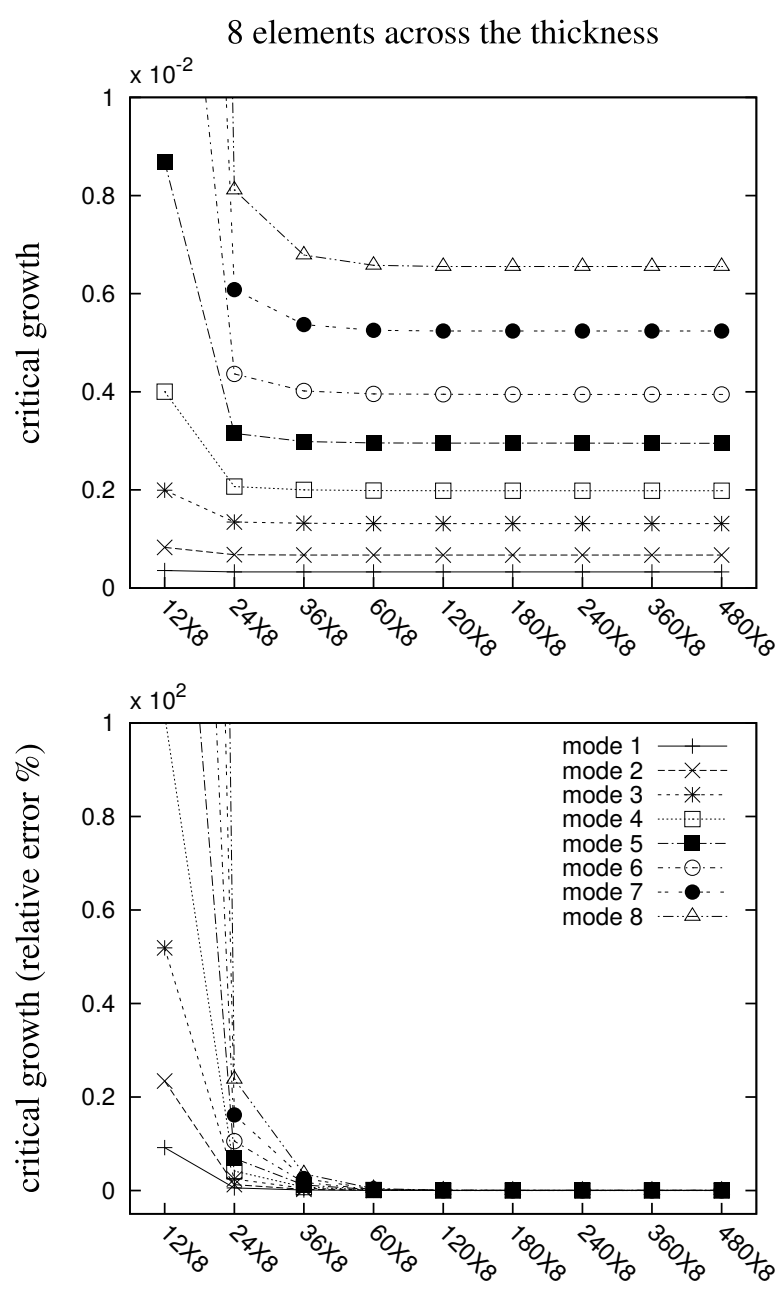

number of elements

Figure 4: The critical growth and the percent error for 8 buckling modes using IGA for various mesh refinements. The $x$-axis shows the number of elements along the beam times the number of element across the height of the beam.
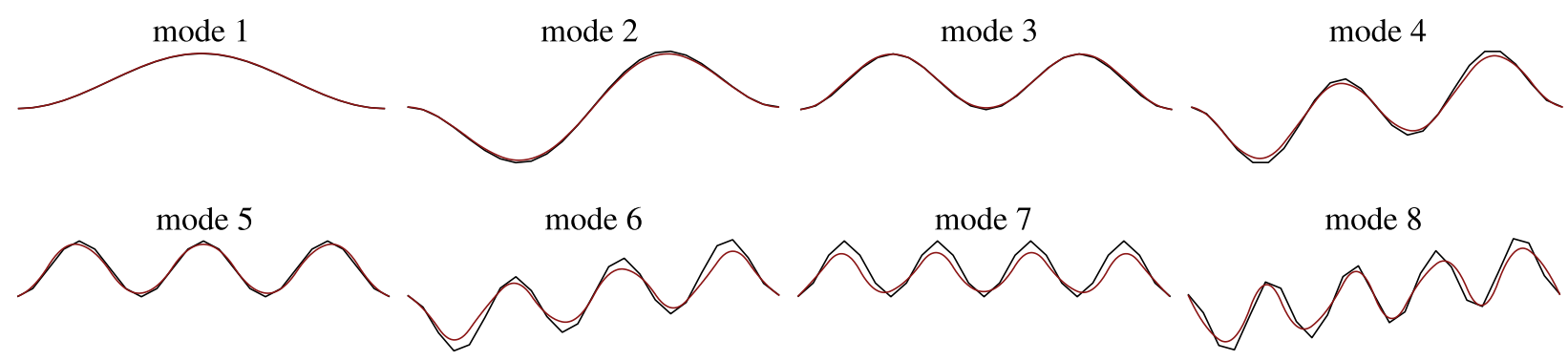

FEA

IGA

Figure 5: The first 8 buckling modes (eigenvectors) of the slender growing beam, comparing the results using FEA (quadratic elements) and IGA (quadratic) both with a coarse mesh. 

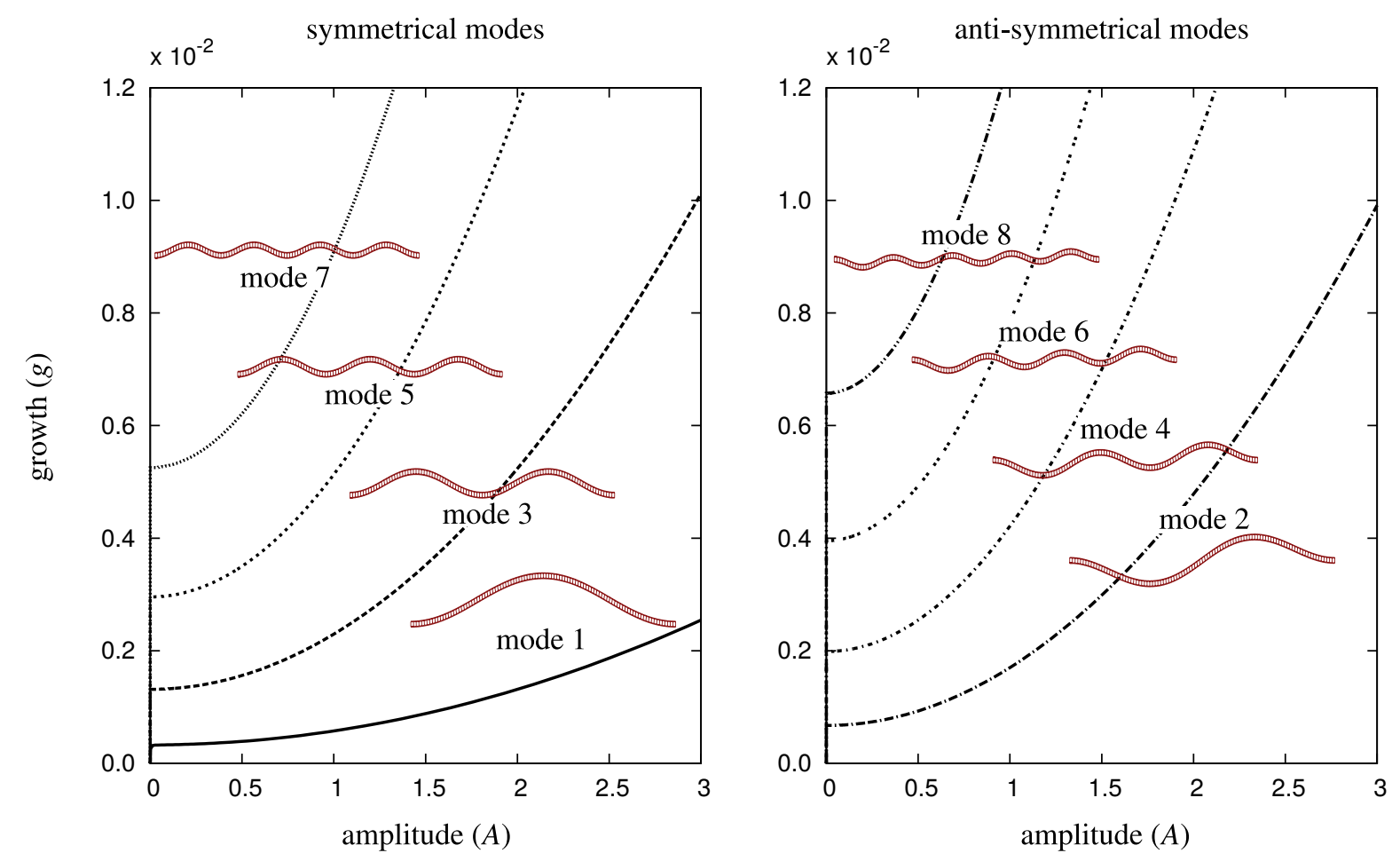

Figure 6: Bifurcation tree of the growing beam using IGA and the deformed shapes obtained by perturbation technique based on the eigenvalue analysis results. The deformed shapes correspond to the growth value of $g=0.02$ and are scaled by a factor of 2 .

and a thin film with the thickness $H_{f}$ perfectly bonded on top of the substrate. The film and the substrate have the Poisson's ratio $v_{f}=v_{s}=0.49$ and we define the stiffness ratio as $\mu_{f} / \mu_{s}$ for later examples.

For simplicity, the overall mesh discretization is defined using a single mesh size parameter $d$, shown in Fig. 7, obtained by dividing the length $L$ by the element numbers in the length direction. For the mesh quality analysis here, $d$ ranges from $d_{\min }$ to $d_{\max }$ for the finest to the coarsest mesh discretizations, respectively. The domain is constructed using two independent patches associated to the film and to the substrate. The patches are connected to each other with $C^{0}$ continuity at NURBS elements with the matching face. The coarsest control net for the problem is shown in the Fig. 7 formed from knot vectors $\Xi=\{0,0,0,1,1,1\}$ and $\mathcal{H}=\{0,0,0,1,1,1\}$, and the polynomial order of the basis functions are set as $p=q=2$ for each patch. Both the film and the substrate grow only along the horizontal axis according to the anisotropic growth tensor $\boldsymbol{F}_{g}=\boldsymbol{I}+g \boldsymbol{e}_{x} \otimes \boldsymbol{e}_{x}$ under plane strain conditions. The growth rates $g_{s}$ and $g_{f}$ pertain to the substrate and to the film, respectively. Throughout the examples in this section, we exclusively assume that the bulk is not growing and fix $g_{s}=0$ from the onset. Note that this assumption does not hold from Sec. 3.3 onward. In the remainder of this section, we first carry out a comparative study of IGA versus FEA to capture wrinkling instabilities in bilayer systems in Sec. 3.2.1. The prime purpose of the analysis is to show that IGA outperforms FEA and justify employing only IGA for all the remaining examples. Next, we compute the critical growth versus stiffness ratio for various film thicknesses in Sec. 3.2.2 using IGA. This example is a typical benchmark for growth-induced instabilities in bilayer systems [79].

\subsubsection{Comparative study of IGA and FEA}

In this section, we compare the performance of IGA with FEA in calculating the onset of growth-induced instabilities, referred to as critical growth, using solely eigenvalue analysis [79] and excluding any perturbation. In order to evaluate the performance of both methods, we perform an h-adaptive sensitivity analysis by varying the mesh size $d$ between $d_{\text {min }} \approx 0.09$ and $d_{\text {max }} \approx 1.86$ and compute the critical growth to initiate the wrinkling instability on the bilayer system of Fig. 7. The problem parameters to define the domain are $L=60, H_{f}=0.8$ and $H_{s}=25$. Figure 8 shows the 


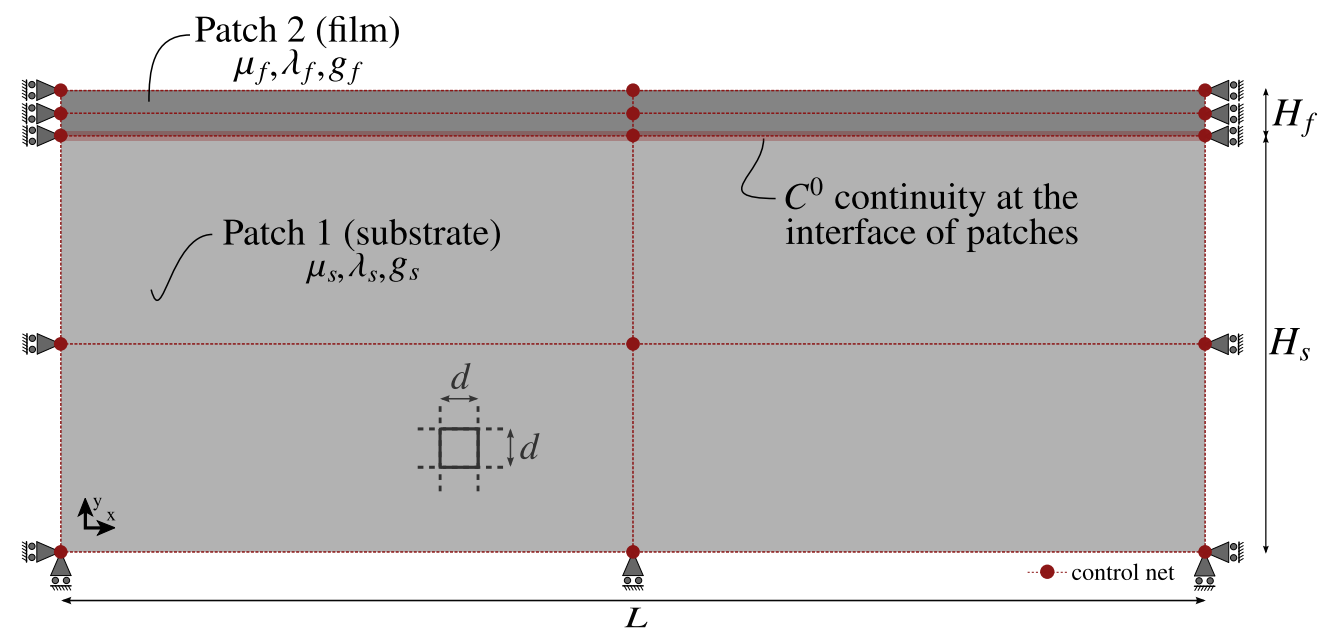

Figure 7: The bilayer system composed of a substrate and a thin film constrained on the sides only in the x-direction and at the bottom only in the $y$-direction. The coarsest control net of bilayer system formed from knot vectors $\Xi=\{0,0,0,1,1,1\}$ and $\mathcal{H}=\{0,0,0,1,1,1\}$, and the polynomial order of $p=q=2$ for each patch. The patches sharing the same edge are connected to each other with $C^{0}$ continuity.
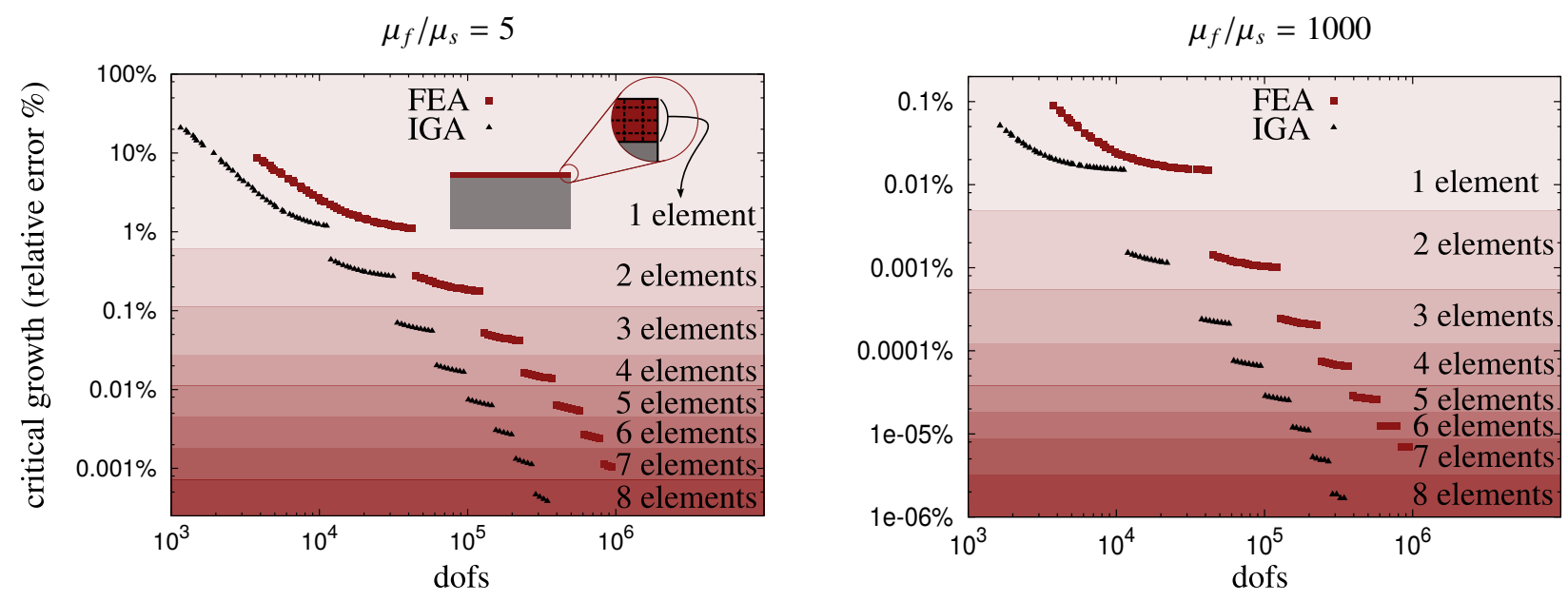

Figure 8: The percent error in the critical growth to initiate wrinkling instability for $\mu_{f} / \mu_{s}=5$ (left) and $\mu_{f} / \mu_{s}=1000$ (right), illustrating the improvement of accuracy with h-adaptive refinement for both IGA and FEA. The results clearly show that IGA furnishes more accurate results for the same number of degrees of freedom compared to FEA. In order to obtain the same accuracy, IGA requires roughly four times less degrees of freedom than FEA. This behavior is qualitatively representative and relevant to other stiffness ratios in this contribution.

convergence of both methods and relative error associated with critical growth with respect to the finest IGA solution for the stiffness ratio $\mu_{f} / \mu_{s}=5$ (left) and $\mu_{f} / \mu_{s}=1000$ (right).

Figure 8 shows that IGA furnishes more accurate results for the same number of degrees of freedom compared to FEA. In order to obtain the same accuracy, IGA requires roughly four times less degrees of freedom than FEA. Furthermore, the obvious jumps to improved solutions reveal the importance of the number of elements across the film thickness. The overall qualitative trends for both stiffness ratios $\mu_{f} / \mu_{s}=5$ (left) and $\mu_{f} / \mu_{s}=1000$ (right) are similar and lead to identical conclusions. However, the errors of the stiffness ratio of 1000 is almost two orders of magnitude less than those of the stiffness ratio 5. This is essentially due to the fact that for larger stiffness ratios, the substrate plays a minor role. In the limit of an extremely high stiffness ratio, the substrate becomes entirely indifferent with respect to the deformation of the film and the bilayer system reduces to the Euler beam. Recall, only the film is growing in this example. 


\subsubsection{Critical growth versus stiffness ratio}

In this section, we devise a systematic numerical analysis to understand the role of stiffness ratio and the film thickness on growth-induced instabilities in bilayer systems. In particular, we study the critical growth and the characteristic wavelength of wrinkling instabilities for a broad range of stiffness ratios $\mu_{f} / \mu_{s}$ and for three film thicknesses of 0.4 , 0.8 and 1.2. All computations are carried out using IGA. Inspired by Fig. 8 of the previous example, we choose the mesh size $d=0.2$. This mesh size corresponds to a relative error of less than $0.01 \%$ for $\mu_{f} / \mu_{s}=5$ thereby the error decreases rapidly for higher stiffness ratios.

Figure 9 assembles all the numerical results from our rigorous eigenvalue analysis [79] suitable for this problem. The critical growth and the number of waves versus the stiffness ratio are gathered in the first and the second column of Fig. 9, respectively. The third column shows the wavelength versus the cubic root of the stiffness ratio demonstrating the ideally linear behavior associated with the analytical solution detailed in Appendix A.2. The numerical analysis reveals that the critical growth is insensitive to the changes in thickness, however, the wavelength decreases by increasing the film thickness. All observations in this analysis agrees well with the analytical solution in Appendix A.2, see also [26] among others.
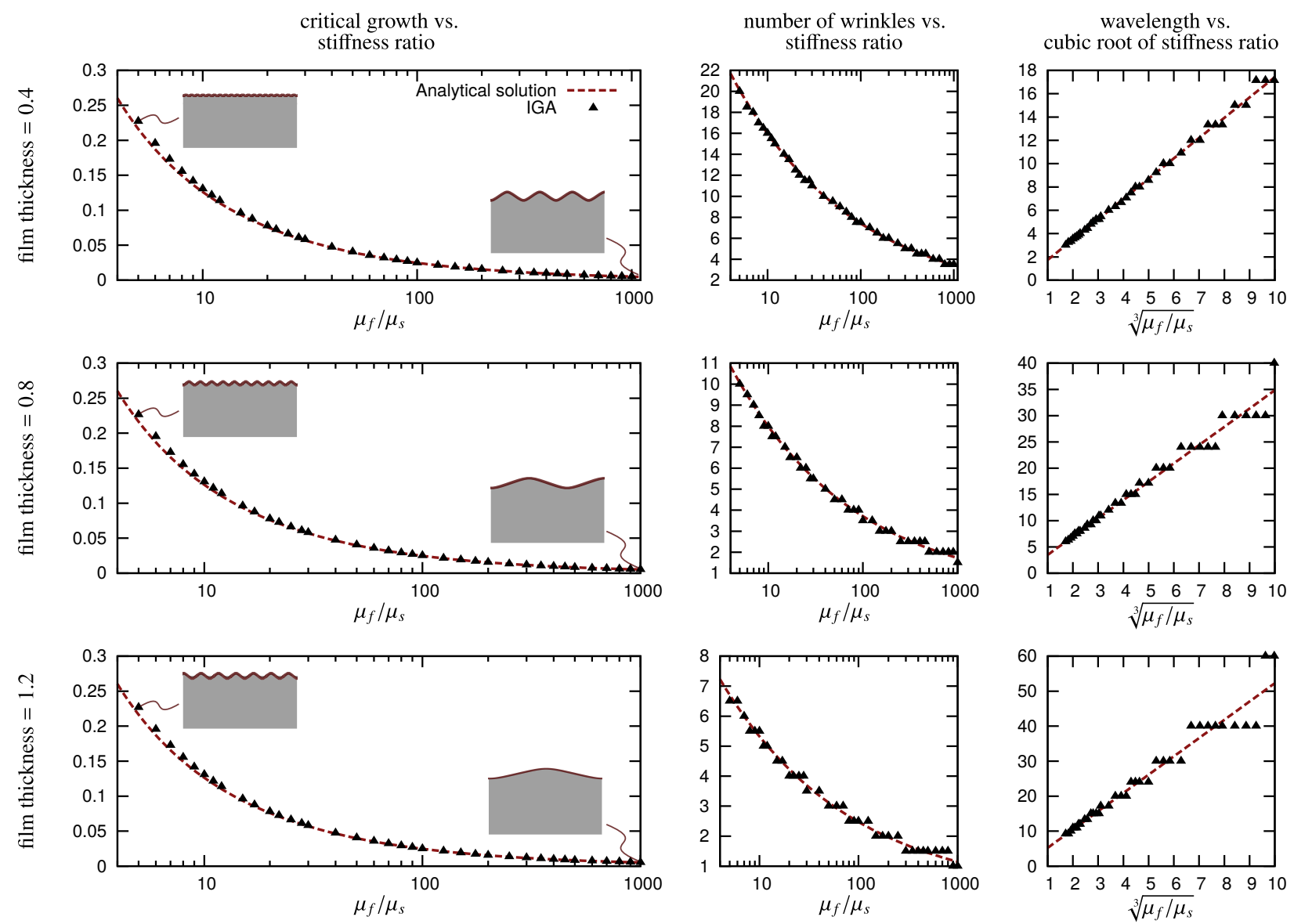

Figure 9: Instability study of a growing film on a deep substrate. The problem parameters are the domain length $L=60$, substrate height $H_{s}=25$ and the film thickness $H_{f}=0.4,0.8,1.2$ with stiffness ratio $5 \leq \mu_{f} / \mu_{s} \leq 1000$. The depicted wrinkled patterns are not the deformed shapes, but the normalized eigenmodes.

\subsection{Period-doubling instability}

So far, we have studied the buckling instabilities in a slender beam due to the growth of the beam along its length in Sec. 3.1. Furthermore, we have elaborated on various aspects of wrinkling instabilities of a bilayer system in Sec. 3.2. 
In both buckling and wrinkling instabilities, we have clearly observed that IGA serves as a powerful tool to capture growth-induced instabilities. In this section, we study a secondary growth-induced instability mode associated with bilayer systems namely, period-doubling. A key feature of period-doubling is that it happens due to the nonlinear response of the substrate. Therefore, we can observe period-doubling only if the substrate undergoes a considerable amount of residual stress. In order to generate compressive stresses in the substrate, we allow that the substrate grows together with the film and hence, in contrast to previous sections, $g_{s} / g_{f}=1$ in this section. Having an identical growth for both the substrate and the film, is qualitatively similar to prescribing a uniform compression on the entire domain from the sides. Clearly, equal compression does not lead to equal stresses in both the substrate and the film. Roughly, the same argument holds for the uniform growth in both domains and thus, justifies its overall comparability to compression. Despite this parallelism between compression and growth, a growing domain is not exactly identical to a domain under compression.

\subsubsection{Understanding the period-doubling through eigenvalue analysis}

In this section, we investigate the secondary instability modes emerging due to further increase of growth in the system and more importantly, due to the growth in both the substrate and the film. In the range of stiffness ratios in this contribution, the secondary instability pattern of interest is period-doubling. Therefore, in the numerical analysis, the domain length $L$ is adjusted to accommodate at least two waves. We have thoroughly explored the influence of the depth of the substrate $H_{s}$ and we are fairly confident that for $H_{s} \geq 3 \lambda_{0}$, we do not observe a noticeable change in the results, whereby $\lambda_{0}$ is the wavelength corresponding to the critical growth for the wrinkling. For the numerical analysis, we set the thickness of the film $H_{f}=0.4$, the domain length $L=2 \lambda_{0}$, the depth of the substrate $H_{s}=3 \lambda_{0}$ and
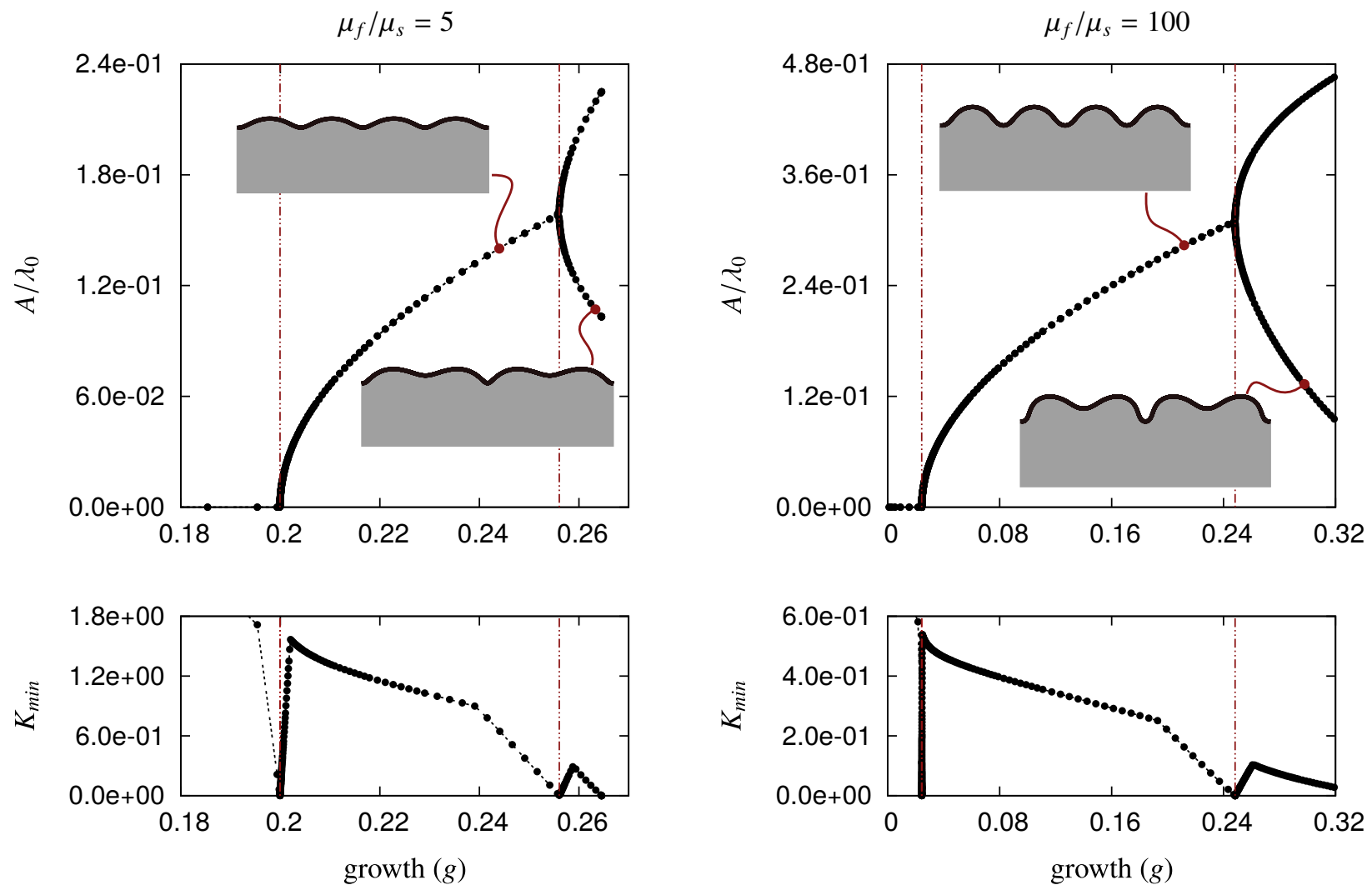

Figure 10: The amplitude growth and the inspection of stability of the system in a increasingly growing bilayer system for stiffness ratio $\mu_{f} / \mu_{s}=5$ (left) and $\mu_{f} / \mu_{s}=100$ (right). The evolution of the normalized amplitude of the characteristic wavelength (top) shall be compared with the smallest eigenvalue (bottom). 
the mesh size $d=0.08$. Note, choosing the domain length as multiples of $2 \lambda$ such as $L=2 \lambda, 4 \lambda, 6 \lambda$ with symmetric boundary conditions does not influence the results at all. This is expected from a theoretical viewpoint and we have also confirmed it numerically although we omit the details of calculations for the sake of brevity.

Eigenvalue analysis makes it possible to capture the onset of wrinkling accurately and efficiently without introducing any perturbations. This technique only determines the onset of the wrinkling instabilities and predicts deformation patterns through associated eigenvectors. In order to analyze the evolution of the actual deformation from flat state to wrinkling and from wrinkling to period-doubling, we need to perturb the systems and follow the formation of different instability patterns. A stress-free displacement is applied on top of the film. The pattern of this displacement is given by $p_{o} \cos \left(2 \pi x / \lambda_{0}\right)$ where $x \in[0, L]$ and the perturbation magnitude factor $p_{o}=10^{-8}$. The need to see the actual formation of different folding patterns should not diminish the importance of eigenvalues though. We constantly use the insight provided through a concurrent eigenvalue analysis to better understand different phenomena as illustrated in Fig. 10.

Figure 10 (top) shows the evolution of amplitude $A$ normalized by the characteristic wavelength $\lambda_{0}$ and the trend of minimum eigenvalue (bottom) for continuously growing confined bilayer. At first, the film disturbed with slight perturbations remains flat under increasing growth prior to the first bifurcation point. The growth within the system accumulates residual stresses until eventually, the film bends together with the substrate and forms sinusoidal wrinkles to release this residual stress. Hence, under a slight increase in compression due to growth at the primary instability point, sinusoidal waves emerge suddenly on the film, forming wrinkled pattern. The amplitudes of waves grow equally until the next instability point. The next instability point defines the onset of period doubling where the periodic waves bifurcate into a pair of growing and decaying waves. Further growth promotes more advanced instability patterns, which fall beyond the scope of this work. Furthermore, we observe that the minimum eigenvalue of the system reaches a local minimum with a value in the range $\left[10^{-3}, 10^{-7}\right]$. The introduced perturbation prevents the system from unstable behavior and the local minima indicate a quantitative prediction of the onset of each instability mode. It is observed that these minima are at the initiations of amplitude growth for wrinkling and pitchfork bifurcation of amplitudes at period-doubling instabilities, which again indicates the importance of eigenvalue analysis alongside the IGA calculations. To capture the instability points within a predefined tolerance, we employ an adaptive time stepping algorithm.

\subsubsection{Phase diagram of growth-induced instabilities}

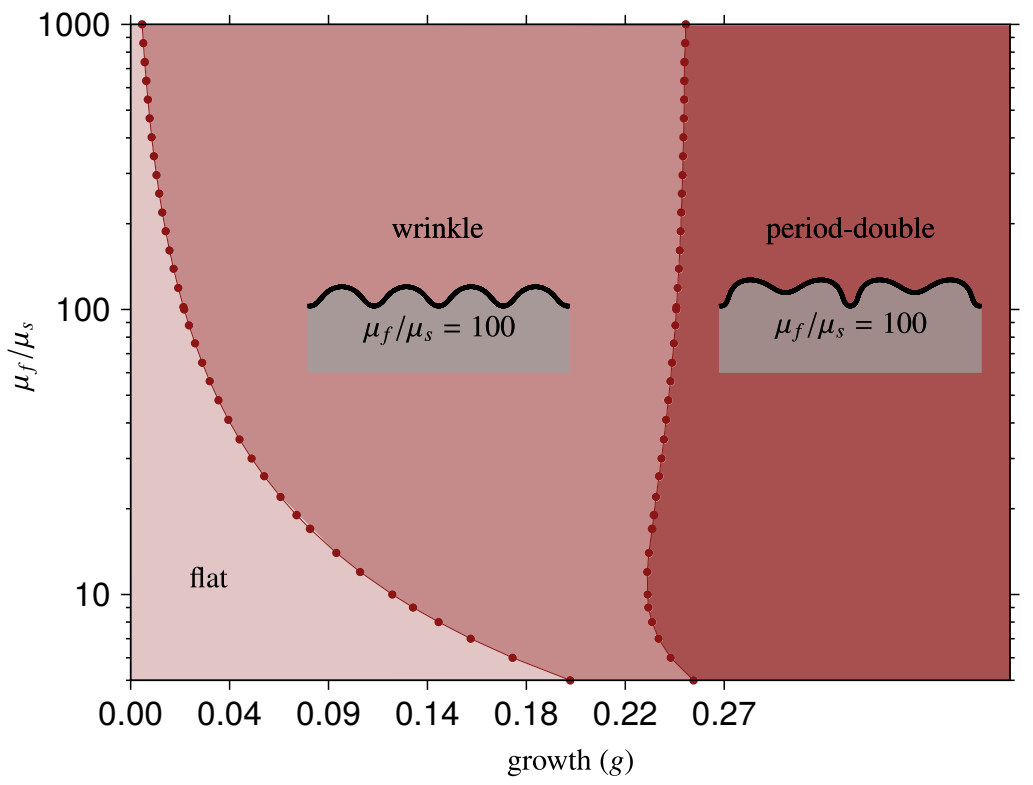

Figure 11: The critical growth values for wrinkling and period-doubling at a increasingly growing bilayer system illustrating the phase transitions for instability modes where $5 \leq \mu_{f} / \mu_{s} \leq 1000$. The deformed shapes are scaled for a better representation. 
We have shown that eigenvalue analysis captures the onset of wrinkling and period-doubling instability through turning points over the minimum eigenvalue trend. By inspection of these points and instability modes, we investigate the evolution of the bilayer system from flat state to wrinkling and, later on, from wrinkling to period-doubling over a broad range of stiffness ratios of $5 \leq \mu_{f} / \mu_{s} \leq 1000$. Figure 11 illustrates a quantitative phase diagram predicting both the wrinkling and period-doubling instability in a growing bilayer system. Through carefully analyzing the results, we can draw the following conclusions. First, the critical growth for wrinkling instability monotonically increases when the stiffness ratio is decreased. This is expected since for a comparatively stiffer film with respect to the substrate, the film needs less growth to overcome the substrate stiffness and to form wrinkles. Second, a significant amount of growth is needed to initiate period-doubling instabilities. For $\mu_{f} / \mu_{s}>60$, the onset of period-doubling is almost insensitive to the stiffness ratio and the critical growth to initiate period-doubling is approximately $g=0.25$. Third, for small stiffness ratios, the gap between the onset of wrinkling and period-doubling instabilities is small and since the wavelength is small in this range, the growth of amplitudes is limited. Hence, residual stress induced by growth is released via the transition from wrinkling to period-doubling. Finally, the growth of both substrate and the film generates enough geometrical and material nonlinearity in the system that leads to period-doubling. In the case of non-growing substrate, we could not observe period-doubling.

\subsection{Influence of growth ratio on instabilities}

So far, we have studied the growth-induced instabilities in bilayer systems for two limit cases of $(i) g_{s} / g_{f}=0$ associated with the non-growing substrate in Sec. 3.2 and (ii) $g_{s} / g_{f}=1$ indicating that the substrate grows equally to the film in Sec. 3.3. The objective of this section is to study the role of the growth ratio between the substrate and the film and cover the whole spectrum of $0 \leq g_{s} / g_{f} \leq 1$. In doing so, the growth ratio is systematically varied in the interval $[0,1]$ and the results are gathered in Fig. 12. The numerical analysis correspond to the stiffness ratio of $\mu_{f} / \mu_{s}=100$ and adopts the isotropic growth for both the film and the substrate.

Figure 12 shows the resulting phase diagram clarifying the influence of growth ratio on the onset of each instability mode such as wrinkling and period-doubling at increasingly growing systems. The numerical findings lead to the following conclusions. First, the onset of wrinkling is insensitive to the variations in growth ratio. This behavior can be explained as follows. Wrinkling phenomena emerge at a flat bilayer system due to a relatively small residual stress.

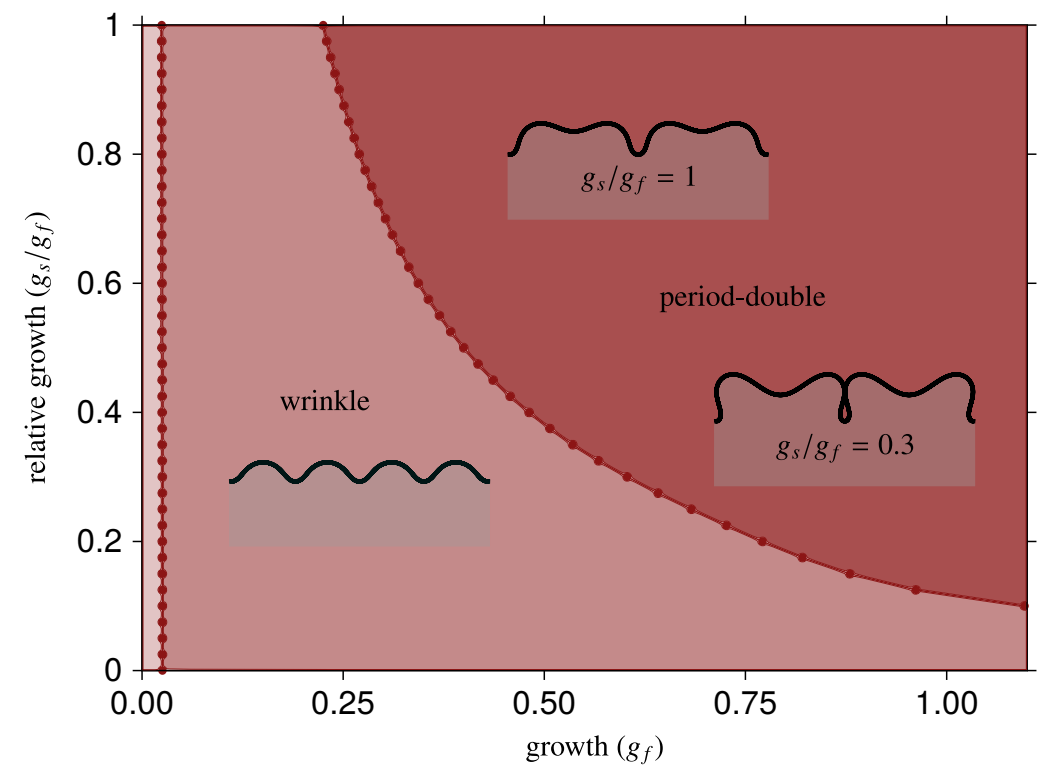

Figure 12: The phase diagram of wrinkling and period-doubling, in which the $y$-axis represents the relative growth and the $\mathrm{x}$-axis is the overall growth at the film in the bilayer systems. For vanishing growth rates at substrate, the secondary instabilities can be delayed and eventually suppressed. 
Hence, the substrate can be considered simply as a linear support for the film, which is in line with the analytical solution in Appendix A.2. Second, and more interestingly, we observe a pronounced delay on the onset of perioddoubling for smaller growth ratios. In particular, the period-doubling cannot be achieved in the limit of a non-growing bulk. Clearly, our findings suggest that the growth of the substrate plays the main role to trigger the period-doubling instabilities in bilayer systems. This can be justified by the fact that the growth of the substrate acts as the underlying mechanism to produce stresses and consequently, to introduce nonlinearities in the material response. Moreover, our results show two distinctive patterns for large and small growth ratios. This occurs because the period-doubling happens at a larger film growth for smaller growth ratios resulting in a more pronounced deformation of the film.

\section{Conclusion}

Understanding morphological instabilities due to compressive stresses in materials has been an extremely interesting topic in the past decade. Morphological instabilities play an important role in living systems as well as in advanced engineering applications. Additionally, computational mechanics has witnessed a significant improvement over the last few years originated in part from the newly introduced method of IGA [24]. Here, we have presented our first attempt to capture morphological instabilities using IGA.

We have established a computational framework to study morphological instabilities, particularly wrinkling and period-doubling. Our framework is essentially IGA enhanced with extra subroutines to carry out eigenvalue analysis. The eigenvalue analysis provides a remarkably useful insight into the instability behavior of the material response. Furthermore, we have compared IGA and FEA and concluded that IGA produces more accurate results for the same number of degrees of freedom. This improvement over the standard FEA together with the ability of IGA to exactly represent the geometry, renders IGA as a superior method to study morphological instabilities of large systems with free-form geometries, see Fig. 13. This established framework is suitable to accommodate different sources of compressive stresses, which result in morphological instabilities, such as mechanical loading, confined thermal loading, and confined or differential material growth/atrophy or swelling/shrinkage.

Our next immediate plan includes the development of efficient path-following algorithms suitable for growthinduced instabilities. In particular, morphological instabilities, such as formation of creases and ridges, require the solution of unstable systems [36,92]. Further extension of this work include contact treatment in the analysis as it plays an important role at highly compressed material systems forming localized instabilities with touching surfaces. Clearly, the current framework is also readily expandable to study instabilities also in trilayer and multi-layer systems $[15,93]$. In summary, this manuscript presents our first attempt to shed light on capturing growth-induced instabilities using IGA. We believe this generic framework is widely applicable to advance the understanding of the emergence and evolution of morphological instabilities with a rich variety of applications in soft material and living systems.
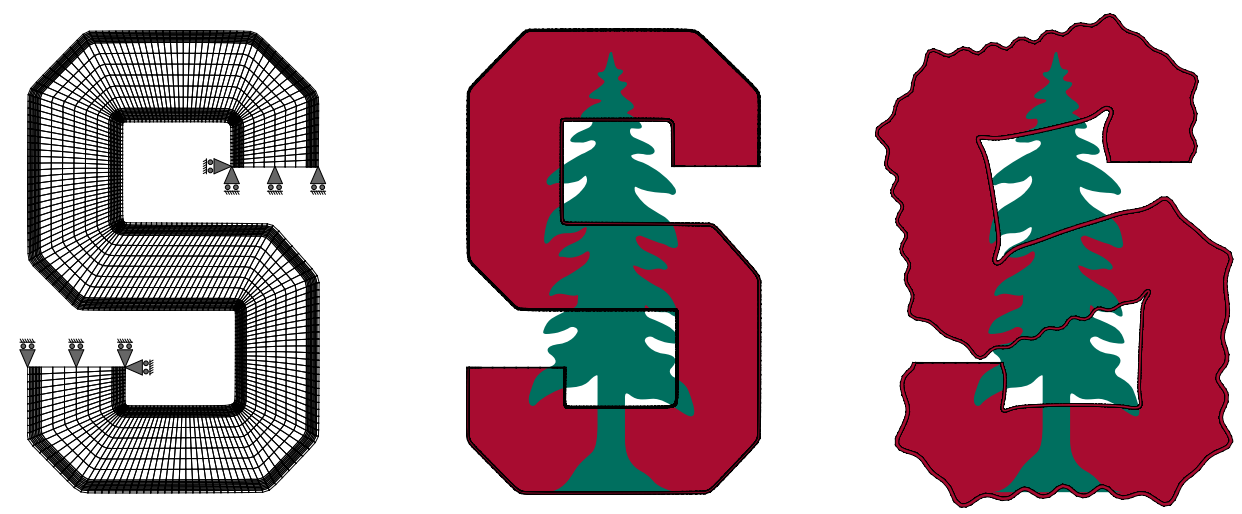

Figure 13: Growth-induced instabilities at free-form geometries, such as the Stanford University Emblem, using IGA. The IGA mesh and boundary conditions with growing outer layer are shown on the left, the undeformed geometry is shown in the center, and the growth induced deformation is shown on the right. 


\section{Appendix A. Analytical solutions for growth-induced instabilities}

The objective of this section is to briefly present the analytical solutions available for growth-induced instabilities. Clearly, the analytical solutions are based on certain simplifying assumptions and are only useful for a very limited number of applications and for verifying the numerical results.

\section{Appendix A.1. Buckling instability of a growing slender beam}

Here, we formulate the analytical solution for the growing slender beam of Sec. 3.1 ideally satisfying the assumptions associated with the Euler beam theory. Consider a slender beam with the anisotropic growth along the beam and clamped on both ends with the prescribed boundary conditions shown in Fig. 3. The governing differential equation for this growing beam with the length $L$ and a square cross section of height $H$ reads

$$
w^{\prime \prime \prime \prime}+\frac{12}{H^{2}} g w^{\prime \prime}=0 \quad \text { where } \quad \text { at } x=0: w=w^{\prime}=0 \quad \text { and } \quad \text { at } x=L: w=w^{\prime}=0 .
$$

Here, $w(x)$ is the deflection in $y$ direction and $g$ indicates the anisotropic growth coefficient. Note that we assume a vanishing Poisson's ratio for simplicity. The boundary conditions are fixed deflections and fixed rotations at both ends. The general solution for the differential equation (A.1) is

$$
w(x)=c_{1} \sin \left(\alpha \frac{x}{L}\right)+c_{2} \cos \left(\alpha \frac{x}{L}\right)+c_{3} x+c_{4} \quad \text { with } \quad \alpha=2 \frac{L}{H} \sqrt{3 g} .
$$

Prescribing the boundary conditions on the general solution (A.2) results in a system of 4 equations and 4 unknowns for which we seek a non-trivial solution. The translational boundary conditions impose $c_{4}=-c_{2}$ and $c_{3}=-c_{1} \alpha / L$ and therefore, reduce the system of equations to two equations as

$$
(\sin \alpha-\alpha) c_{1}+(\cos \alpha-1) c_{2}=0 \quad \text { and } \quad(\cos \alpha-1) c_{1}+(-\sin \alpha) c_{2}=0 .
$$

In order to obtain the non-trivial solution for the coefficients $c_{1}$ and $c_{2}$, we construct a matrix of coefficients and set its determinant to zero which result in one non-linear equation

$$
\alpha \sin (\alpha)+2 \cos (\alpha)-2 \stackrel{!}{=} 0
$$

or alternatively

$$
\sin \left(\frac{\alpha}{2}\right)\left[\alpha \cos \left(\frac{\alpha}{2}\right)-2 \sin \left(\frac{\alpha}{2}\right)\right] \stackrel{!}{=} 0 .
$$

Therefore, the solutions of (A.5) are

$$
\sin \left(\frac{\alpha}{2}\right) \stackrel{!}{=} 0 \quad \Rightarrow \quad \alpha=2 \pi, 4 \pi, \ldots \quad \text { and } \quad \tan \left(\frac{\alpha}{2}\right) \stackrel{!}{=} \frac{\alpha}{2} \quad \Rightarrow \quad \alpha=8.9868,15.4505, \ldots
$$

The solutions (A.6) render the critical growth values via the relation

$$
g_{c}=\frac{1}{12}\left(\frac{H}{L}\right)^{2} \alpha^{2}
$$

from (A.2). For each critical $\alpha$ the particular solution of the problem reduces to

$$
\frac{w(x)}{c_{2}}=\frac{1-\cos \alpha}{\sin \alpha-\alpha}\left[\sin \left(\alpha \frac{x}{L}\right)-\left(\alpha \frac{x}{L}\right)\right]+\cos \left(\alpha \frac{x}{L}\right)-1 .
$$

For a detailed exposition on elastic stability of beams, see [94]. 


\section{Appendix A.2. Wrinkling instability of a growing film on a compliant substrate}

In this section we formulate the analytical solution for the classical bilayer system with a thin film adhered to the infinite half-space substrate. We consider the system under plane-strain conditions. The governing differential equation for the film under a distributed load $q$ applied to the film from the elastic substrate is

$$
\frac{\bar{E}_{f} H_{f}^{3}}{12} w^{\prime \prime \prime \prime}+P H_{f} w^{\prime \prime}=q
$$

in which $\bar{E}_{f}$ denotes the plane strain elastic modulus of the film related to the elastic modulus $E_{f}$ and the Poisson's ratio $v_{f}$ of the film according to $\bar{E}_{f}=E_{f} /\left(1-v_{f}^{2}\right)$. The thickness of the film is denoted $H_{f}$ and $P$ is the axial pressure induced in the film. The general solution of (A.9) is of the form $w=w_{0} \cos (n x)$ in which $n$ is the wave number and $w_{0}$ is the amplitude of the wave. The key step to proceed is that a sinusoidal deflection on top of an infinite substrate results exactly in a sinusoidal reaction with the same wavelength but different amplitude [27, 28]. This follows immediately using the Airy's stress functions on an infinite half-space but, we omit the detailed derivations for this step. Therefore, the transversal load $q$ exerted on the film takes the form

$$
q=-\frac{2 \bar{E}_{s} n}{\zeta} w_{0} \cos (n x) \quad \text { with } \quad \zeta=\frac{3-4 v_{s}}{\left(1-v_{s}\right)^{2}}
$$

in which the plane strain elastic modulus of the substrate is defined as $\bar{E}_{s}=E_{s} /\left(1-v_{s}^{2}\right)$ with $E_{s}$ and $v_{s}$ are the modulus of elasticity and the Poisson's ratio of the substrate, respectively. Substituting (A.10) into (A.9) and making use of the second and fourth derivative of the deflection $w$, the expression for the axial pressure $P$ in the film reads

$$
P=\bar{E}_{f} \frac{H_{f}^{2}}{12} n^{2}+\frac{2 \bar{E}_{s}}{\zeta n H_{f}} .
$$

The wrinkling instability initiates at a critical wave number $n_{\text {crit }}$ at which $\mathrm{d} P / \mathrm{d} n=0$. Hence, taking the derivative of (A.11) with respect to the wave number $n$, and finding its roots, we obtain the critical wave number and the wavelength as

$$
n_{\text {crit }}=\frac{1}{H_{f}} \sqrt[3]{\frac{12 \bar{E}_{s}}{\zeta \bar{E}_{f}}} \quad \text { and } \quad \lambda_{\text {crit }}=2 \pi H_{f} \sqrt[3]{\frac{\zeta \bar{E}_{f}}{12 \bar{E}_{s}}} \quad \text { with } \quad \lambda_{\text {crit }}=2 \pi / n_{\text {crit }} .
$$

The critical strain $\varepsilon_{c r i t}$ can be derived by making use of the linear relation $P=\bar{E}_{f} \varepsilon$ and substituting (A.12) into (A.11) as

$$
\varepsilon_{c r i t}=\left(\frac{3 \bar{E}_{s}}{2 \zeta \bar{E}_{f}}\right)^{2 / 3} \Rightarrow g_{c r i t}=\frac{\varepsilon_{c r i t}}{1-\varepsilon_{c r i t}} .
$$

The derivation of the interrelation between the growth $g$ and the overall strain $\varepsilon$ is straightforward and reads through the linearization of the growth tensor $\boldsymbol{F}_{g}=(1+g) \boldsymbol{I}-g \boldsymbol{N}_{g} \otimes \boldsymbol{N}_{g}$ as $\varepsilon=g /(1+g)$.

\section{Acknowledgements}

Financial support was provided by the National Science Foundation through CAREER Award CMMI-1553638 and the James M. Gere Research Fellowship from Stanford University. This support is gratefully acknowledged.

\section{References}

[1] B. Li, Y.-P. Cao, X.-Q. Feng, H. Gao, Mechanics of morphological instabilities and surface wrinkling in soft materials: a review, Soft Matter 8 (2012) 5728-5745.

[2] Q. Wang, X. Zhao, Beyond wrinkles: Multimodal surface instabilities for multifunctional patterning, MRS Bulletin 41 (2016) $115-122$.

[3] Q. Wang, X. Zhao, A three-dimensional phase diagram of growth-induced surface instabilities., Scientific Reports 5 (2015) 8887. 
[4] N. Hu, R. Burgueño, Buckling-induced smart applications: recent advances and trends, Smart Materials and Structures 24 (2015) 63001.

[5] S. Yang, K. Khare, P.-C. Lin, Harnessing surface wrinkle patterns in soft matter, Advanced Functional Materials 20 (2010) $2550-2564$.

[6] J. A. Rogers, T. Someya, Y. Huang, Materials and mechanics for stretchable electronics, Science 327 (2010) $1603-1607$.

[7] S. Xu, Z. Yan, K.-I. Jang, W. Huang, H. Fu, J. Kim, Z. Wei, M. Flavin, J. McCracken, R. Wang, A. Badea, Y. Liu, D. Xiao, G. Zhou, J. Lee, H. U. Chung, H. Cheng, W. Ren, A. Banks, X. Li, U. Paik, R. G. Nuzzo, Y. Huang, Y. Zhang, J. A. Rogers, Assembly of micro/nanomaterials into complex, three-dimensional architectures by compressive buckling, Science 347 (2015) 154-159.

[8] J. Genzer, J. Groenewold, Soft matter with hard skin: From skin wrinkles to templating and material characterization, Soft Matter 2 (2006) 310-323.

[9] C.-H. Li, C. Wang, C. Keplinger, J.-L. Zuo, L. Jin, Y. Sun, P. Zheng, Y. Cao, F. Lissel, C. Linder, X.-Z. You, Z. Bao, A highly stretchable autonomous self-healing elastomer, Nature Chemistry 8 (2016) 618-624.

[10] D. P. Richman, R. M. Stewart, J. W. Hutchinson, V. S. Caviness, Mechanical model of brain convolutional development, Science 189 (1975) $18-21$.

[11] E. Kuhl, Biophysics: Unfolding the brain, Nature Physics (2016).

[12] S. Budday, P. Steinmann, E. Kuhl, Secondary instabilities modulate cortical complexity in the mammalian brain, Philosophical Magazine (2015) 37-41.

[13] E. Lejeune, A. Javili, J. Weickenmeier, E. Kuhl, C. Linder, Tri-layer wrinkling as a mechanism for anchoring center initiation in the developing cerebellum, Soft Matter 12 (2016) 5613-5620.

[14] A. B. Tepole, C. J. Ploch, J. Wong, A. K. Gosain, E. Kuhl, Growing skin: A computational model for skin expansion in reconstructive surgery, Journal of the Mechanics and Physics of Solids 59 (2011) 2177-2190.

[15] E. Lejeune, A. Javili, C. Linder, An algorithmic approach to multi-layer wrinkling, Extreme Mechanics Letters 7 (2016) $10-17$.

[16] P. Ciarletta, V. Balbi, E. Kuhl, Pattern selection in growing tubular tissues, Physical Review Letters 113 (2014).

[17] M. Eskandari, M. R. Pfaller, E. Kuhl, On the role of mechanics in chronic lung disease, Materials 6 (2013) 5639.

[18] J. N. Wilking, V. Zaburdaev, M. De Volder, R. Losick, M. P. Brenner, D. a. Weitz, Liquid transport facilitated by channels in Bacillus subtilis biofilms., Proceedings of the National Academy of Sciences of the United States of America 110 (2013) 848-52.

[19] M. Trejo, C. Douarche, V. Bailleux, C. Poulard, S. Mariot, C. Regeard, E. Raspaud, Elasticity and wrinkled morphology of Bacillus subtilis pellicles., Proceedings of the National Academy of Sciences of the United States of America 110 (2013) 2011-6.

[20] D. Y. Khang, J. A. Rogers, H. H. Lee, Mechanical buckling: Mechanics, metrology, and stretchable electronics, Advanced Functional Materials 19 (2009) 1526-1536.

[21] E. P. Chan, E. J. Smith, R. C. Hayward, A. J. Crosby, Surface wrinkles for smart adhesion, Advanced Materials 20 (2008) $711-716$.

[22] C. M. Stafford, C. Harrison, K. L. Beers, A. Karim, E. J. Amis, M. R. VanLandingham, H. C. Kim, W. Volksen, R. D. Miller, E. E. Simonyi, A buckling-based metrology for measuring the elastic moduli of polymeric thin films, Nature Materials 3 (2004) 545-550.

[23] D. Terwagne, M. Brojan, P. M. Reis, Smart morphable surfaces for aerodynamic drag control, Advanced Materials 26 (2014) $6608-6611$.

[24] T. Hughes, J. Cottrell, Y. Bazilevs, Isogeometric analysis: CAD, finite elements, NURBS, exact geometry and mesh refinement, Computer Methods in Applied Mechanics and Engineering 194 (2005) 4135-4195.

[25] K. Efimenko, M. Rackaitis, E. Manias, A. Vaziri, L. Mahadevan, J. Genzer, Nested self-similar wrinkling patterns in skins., Nature Materials 4 (2005) 293-7.

[26] H. Mei, C. M. Landis, R. Huang, Concomitant wrinkling and buckle-delamination of elastic thin films on compliant substrates, Mechanics of Materials 43 (2011) 627-642.

[27] M. A. Biot, Folding Instability of a Layered Viscoelastic Medium under Compression, Proceedings of the Royal Society A: Mathematical, Physical and Engineering Sciences 242 (1957) 444-454.

[28] H. G. Allen, Analysis and Design of Structural Sandwich Panels, Elsevier, 1969.

[29] J. W. Hutchinson, The role of nonlinear substrate elasticity in the wrinkling of thin films, Philosophical Transactions of the Royal Society A: Mathematical, Physical and Engineering Sciences 371 (2013) 20120422

[30] Z. Y. Huang, W. Hong, Z. Suo, Nonlinear analyses of wrinkles in a film bonded to a compliant substrate, Journal of the Mechanics and Physics of Solids 53 (2005) 2101-2118.

[31] Y. Cao, J. W. Hutchinson, Wrinkling phenomena in neo-hookean film/substrate bilayers, Journal of Applied Mechanics 79 (2012) 031019031019.

[32] J. Song, H. Jiang, Z. Liu, D. Khang, Y. Huang, J. Rogers, C. Lu, C. Koh, Buckling of a stiff thin film on a compliant substrate in large deformation, International Journal of Solids and Structures 45 (2008) 3107-3121.

[33] L. Jin, A. Takei, J. W. Hutchinson, Mechanics of wrinkle/ridge transitions in thin film/substrate systems, Journal of the Mechanics and Physics of Solids 81 (2015) 22-40.

[34] F. Brau, P. Damman, H. Diamant, T. A. Witten, Wrinkle to fold transition: influence of the substrate response, Soft Matter 9 (2013) 8177.

[35] T. Tallinen, J. S. Biggins, L. Mahadevan, Surface sulci in squeezed soft solids, Physical Review Letters 110 (2013) 024302.

[36] M. Diab, T. Zhang, R. Zhao, H. Gao, K.-S. Kim, Ruga mechanics of creasing: from instantaneous to setback creases, Proceedings of the Royal Society A: Mathematical, Physical and Engineering Sciences 469 (2013) 20120753.

[37] J. Zang, X. Zhao, Y. Cao, J. W. Hutchinson, Localized ridge wrinkling of stiff films on compliant substrates, Journal of the Mechanics and Physics of Solids 60 (2012) 1265-1279.

[38] F. Brau, H. Vandeparre, A. Sabbah, C. Poulard, A. Boudaoud, P. Damman, Multiple-length-scale elastic instability mimics parametric resonance of nonlinear oscillators, Nature Physics 7 (2011) 56-60.

[39] R. Zhao, T. Zhang, M. Diab, H. Gao, K.-S. Kim, The primary bilayer ruga-phase diagram I: Localizations in ruga evolution, Extreme Mechanics Letters 4 (2015) 76-82.

[40] J.-Y. Sun, S. Xia, M.-W. Moon, K. H. Oh, K.-S. Kim, Folding wrinkles of a thin stiff layer on a soft substrate, Proceedings of the Royal Society A: Mathematical, Physical and Engineering Sciences 468 (2012) 932-953.

[41] S. Budday, E. Kuhl, J. W. Hutchinson, Period-doubling and period-tripling in growing bilayered systems, Philosophical Magazine 95 (2015) $3208-3224$ 
[42] Y. Zhao, Y. Cao, W. Hong, M. K. Wadee, X.-Q. Feng, Towards a quantitative understanding of period-doubling wrinkling patterns occurring in film/substrate bilayer systems, Proceedings of the Royal Society A 471 (2015) 20140695

[43] S. Tang, Y. Li, Y. Yang, Z. Guo, The effect of mechanical-driven volumetric change on instability patterns of bilayered soft solids, Soft Matter 11 (2015) 7911-7919.

[44] J. Liu, K. Bertoldi, Bloch wave approach for the analysis of sequential bifurcations in bilayer structures, Proceedings of the Royal Society of London A: Mathematical, Physical and Engineering Sciences 471 (2015).

[45] J. Cottrell, T. Hughes, A. Reali, Studies of refinement and continuity in isogeometric structural analysis, Computer Methods in Applied Mechanics and Engineering 196 (2007) 4160-4183.

[46] Y. Bazilevs, L. Beirão Da Veiga, J. A. Cottrell, T. J. R. Hughes, G. Sangalli, Isogeometric analysis: Approximation, stability and error estimates for h-refined meshes, Mathematical Models and Methods in Applied Sciences 16 (2006) 1031-1090.

[47] V. P. Nguyen, C. Anitescu, S. P. Bordas, T. Rabczuk, Isogeometric analysis: An overview and computer implementation aspects, Mathematics and Computers in Simulation 117 (2015) 89-116.

[48] J. A. Cottrell, T. J. R. Hughes, Y. Bazilevs, Isogeometric Analysis: Toward Integration of CAD and FEA, Wiley Publishing, 1 st edition, 2009.

[49] K. A. Johannessen, T. Kvamsdal, T. Dokken, Isogeometric analysis using LR B-splines, Computer Methods in Applied Mechanics and Engineering 269 (2014) 471-514.

[50] M. R. Dörfel, B. Jüttler, B. Simeon, Adaptive isogeometric analysis by local h-refinement with T-splines, Computer Methods in Applied Mechanics and Engineering 199 (2010) 264-275.

[51] T. W. Sederberg, D. L. Cardon, G. T. Finnigan, N. S. North, J. Zheng, T. Lyche, T-spline simplification and local refinement, ACM Transactions on Graphics 23 (2004) 276.

[52] T. W. Sederberg, J. Zheng, A. Bakenov, A. Nasri, T-splines and T-NURCCs, ACM Transactions on Graphics 22 (2003) 477.

[53] Y. Bazilevs, V. Calo, J. Cottrell, J. Evans, T. Hughes, S. Lipton, M. Scott, T. Sederberg, Isogeometric analysis using T-splines, Computer Methods in Applied Mechanics and Engineering 199 (2010) 229-263.

[54] M. Scott, X. Li, T. Sederberg, T. Hughes, Local refinement of analysis-suitable T-splines, Computer Methods in Applied Mechanics and Engineering 213 (2012) 206-222.

[55] P. Wang, J. Xu, J. Deng, F. Chen, Adaptive isogeometric analysis using rational PHT-splines, Computer-Aided Design 43 (2011) $1438-1448$.

[56] D. Schillinger, L. Dedè, M. A. Scott, J. A. Evans, M. J. Borden, E. Rank, T. J. Hughes, An isogeometric design-through-analysis methodology based on adaptive hierarchical refinement of NURBS, immersed boundary methods, and T-spline CAD surfaces, Computer Methods in Applied Mechanics and Engineering 249-252 (2012) 116-150.

[57] A.-V. Vuong, C. Giannelli, B. Jüttler, B. Simeon, A hierarchical approach to adaptive local refinement in isogeometric analysis, Computer Methods in Applied Mechanics and Engineering 200 (2011) 3554-3567.

[58] X. Wei, Y. J. Zhang, T. J. Hughes, M. A. Scott, Extended Truncated Hierarchical Catmull-Clark Subdivision, Computer Methods in Applied Mechanics and Engineering 299 (2016) 316-336.

[59] X. Li, F. Chen, H. Kang, J. Deng, A survey on the local refinable splines, Science China Mathematics 59 (2016) 617-644.

[60] F. Auricchio, M. Conti, M. Ferraro, S. Morganti, A. Reali, R. Taylor, Innovative and efficient stent flexibility simulations based on isogeometric analysis, Computer Methods in Applied Mechanics and Engineering 295 (2015) 347-361.

[61] S. Morganti, F. Auricchio, D. Benson, F. Gambarin, S. Hartmann, T. Hughes, A. Reali, Patient-specific isogeometric structural analysis of aortic valve closure, Computer Methods in Applied Mechanics and Engineering 284 (2015) 508-520.

[62] S. Lipton, J. Evans, Y. Bazilevs, T. Elguedj, T. Hughes, Robustness of isogeometric structural discretizations under severe mesh distortion, Computer Methods in Applied Mechanics and Engineering 199 (2010) 357-373.

[63] T. Hughes, A. Reali, G. Sangalli, Duality and unified analysis of discrete approximations in structural dynamics and wave propagation: Comparison of p-method finite elements with k-method NURBS, Computer Methods in Applied Mechanics and Engineering 197 (2008) 4104-4124.

[64] A. B. Tepole, M. Gart, A. K. Gosain, E. Kuhl, Characterization of living skin using multi-view stereo and isogeometric analysis, Acta Biomaterialia 10 (2014) 4822-4831.

[65] L. Chen, N. Nguyen-Thanh, H. Nguyen-Xuan, T. Rabczuk, S. P. A. Bordas, G. Limbert, Explicit finite deformation analysis of isogeometric membranes, Computer Methods in Applied Mechanics and Engineering 277 (2014) 104-130.

[66] I. Temizer, Multiscale thermomechanical contact: Computational homogenization with isogeometric analysis, International Journal for Numerical Methods in Engineering 97 (2014) 582-607.

[67] C. V. Verhoosel, M. A. Scott, T. J. R. Hughes, R. de Borst, An isogeometric analysis approach to gradient damage models, International Journal for Numerical Methods in Engineering 86 (2011) 115-134.

[68] S. Rudraraju, A. der Ven, K. Garikipati, Three-dimensional isogeometric solutions to general boundary value problems of Toupin's gradient elasticity theory at finite strains, Computer Methods in Applied Mechanics and Engineering 278 (2014) 705-728.

[69] A. Embar, J. Dolbow, I. Harari, Imposing dirichlet boundary conditions with Nitsche's method and spline-based finite elements, International Journal for Numerical Methods in Engineering 83 (2010) 877-898.

[70] H. Gómez, V. M. Calo, Y. Bazilevs, T. J. R. Hughes, Isogeometric analysis of the Cahn-Hilliard phase-field model, Computer Methods in Applied Mechanics and Engineering 197 (2008) 4333-4352.

[71] J. Kiendl, K.-U. Bletzinger, J. Linhard, R. Wüchner, Isogeometric shell analysis with Kirchhoff-Love elements, Computer Methods in Applied Mechanics and Engineering 198 (2009) 3902-3914.

[72] A. Reali, An Isogeometric Analysis approach for the study of structural vibrations, Journal of Earthquake Engineering 10 (2006) 1-30.

[73] M. A. Scott, M. J. Borden, C. V. Verhoosel, T. W. Sederberg, T. J. R. Hughes, Isogeometric finite element data structures based on Bézier extraction of T-splines, International Journal for Numerical Methods in Engineering 88 (2011) 126-156.

[74] I. Temizer, P. Wriggers, T. J. R. Hughes, Contact treatment in isogeometric analysis with NURBS, Computer Methods in Applied Mechanics and Engineering 200 (2011) 1100-1112.

[75] W. A. Wall, M. A. Frenzel, C. Cyron, Isogeometric structural shape optimization, Computer Methods in Applied Mechanics and Engineering 197 (2008) 2976-2988. 
[76] L. De Lorenzis, I. Temizer, P. Wriggers, G. Zavarise, A large deformation frictional contact formulation using NURBS-based isogeometric analysis, International Journal for Numerical Methods in Engineering 87 (2011) 1278-1300.

[77] C. V. Verhoosel, M. A. Scott, R. De Borst, T. J. R. Hughes, An isogeometric approach to cohesive zone modeling, International Journal for Numerical Methods in Engineering 87 (2011) 336-360.

[78] A. Buffa, G. Sangalli, R. Vázquez, Isogeometric analysis in electromagnetics: B-splines approximation, Computer Methods in Applied Mechanics and Engineering 199 (2010) 1143-1152.

[79] A. Javili, B. Dortdivanlioglu, E. Kuhl, C. Linder, Computational aspects of growth-induced instabilities through eigenvalue analysis, Computational Mechanics 56 (2015) 405-420.

[80] E. K. Rodriguez, A. Hoger, A. D. McCulloch, Stress-dependent finite growth in soft elastic tissues, Journal of Biomechanics 27 (1994) 455 $-467$.

[81] L. A. Taber, Biomechanics of Growth, Remodeling, and Morphogenesis, Applied Mechanics Reviews 48 (1995) 487.

[82] M. Epstein, G. A. Maugin, Thermomechanics of volumetric growth in uniform bodies, International Journal of Plasticity 16 (2000) 951-978.

[83] S. Göktepe, O. J. Abilez, E. Kuhl, A generic approach towards finite growth with examples of athlete's heart, cardiac dilation, and cardiac wall thickening, Journal of the Mechanics and Physics of Solids 58 (2010) 1661-1680.

[84] D. Ambrosi, G. A. Ateshian, E. M. Arruda, S. C. Cowin, J. Dumais, A. Goriely, G. A. Holzapfel, J. D. Humphrey, R. Kemkemer, E. Kuhl, J. E. Olberding, L. A. Taber, K. Garikipati, Perspectives on biological growth and remodeling, Journal of the Mechanics and Physics of Solids 59 (2011) 863-883.

[85] H. Demirkoparan, T. J. Pence, H. Tsai, Hyperelastic internal balance by multiplicative decomposition of the deformation dradient, Archive for Rational Mechanics and Analysis 214 (2014) 923-970.

[86] A. Javili, P. Steinmann, E. Kuhl, A novel strategy to identify the critical conditions for growth-induced instabilities, Journal of the Mechanical Behavior of Biomedical Materials 29 (2014) 20-32.

[87] A. Menzel, E. Kuhl, Frontiers in growth and remodeling, Mechanics Research Communications 42 (2012) 1-14. Recent Advances in the Biomechanics of Growth and Remodeling.

[88] C. Linder, M. Tkachuk, C. Miehe, A micromechanically motivated diffusion-based transient network model and its incorporation into finite rubber viscoelasticity, Journal of the Mechanics and Physics of Solids 59 (2011) 2134-2156.

[89] M. Tkachuk, C. Linder, The maximal advance path constraint for the homogenization of materials with random network microstructure, Philosophical Magazine 92 (2012) 2779-2808

[90] A. Raina, C. Linder, A homogenization approach for nonwoven materials based on fiber undulations and reorientation, Journal of the Mechanics and Physics of Solids 65 (2014) 12-34.

[91] L. Piegl, W. Tiller, The NURBS book, Springer, Berlin, 2nd edition, 1997.

[92] L. Jin, A. Auguste, R. C. Hayward, Z. Suo, Bifurcation diagrams for the formation of wrinkles or creases in soft bilayers, Journal of Applied Mechanics 82 (2015) 061008

[93] E. Lejeune, A. Javili, C. Linder, Understanding geometric instabilities in thin films via a multi-layer model, Soft Matter 12 (2016) 806

[94] S. P. Timoshenko, J. M. Gere, Theory of Elastic Stability, McGraw-Hill, 1961. 